\title{
On Topological Analysis of Entropy Measures for Silicon Carbides Networks
}

\author{
Xing-Long Wang, ${ }^{1}$ Muhammad Kamran Siddiqui $\left(\mathbb{D},{ }^{2}\right.$ Syed Ajaz K. Kirmani, ${ }^{3}$ \\ Shazia Manzoor (D), ${ }^{2}$ Sarfraz Ahmad (iD), ${ }^{2}$ and Mlamuli Dhlamini (iD) \\ ${ }^{1}$ Department of General Education, Anhui Xinhua University, Hefei 230000, China \\ ${ }^{2}$ Department of Mathematics, COMSATS University Islamabad, Lahore Campus, Lahore, Pakistan \\ ${ }^{3}$ Department of Electrical Engineering, College of Engineering, Qassim University, Unaizah, Saudi Arabia \\ ${ }^{4}$ Department of Applied Mathematics, National University of Science and Technology, Bulawayo, Zimbabwe
}

Correspondence should be addressed to Mlamuli Dhlamini; mlamuli.dhlamini@nust.ac.zw

Received 1 September 2021; Accepted 22 October 2021; Published 5 November 2021

Academic Editor: Xuzhen Zhu

Copyright (c) 2021 Xing-Long Wang et al. This is an open access article distributed under the Creative Commons Attribution License, which permits unrestricted use, distribution, and reproduction in any medium, provided the original work is properly cited.

\begin{abstract}
The silicon material has provoked and stimulated significant research concern to a considerable extent taking into account its marvelous mechanical, optical, and electronic properties. Naturally, silicons are semiconductors and are utilized in the formation of various materials. For example, it is used in assembling the electronic based gadgets. In this article, we have studied the 2D structure of silicon carbide $\mathrm{Si}_{2} \mathrm{C}_{3}-I[m, n]$ and $\mathrm{Si}_{2} \mathrm{C}_{3}-I I[m, n]$ and then continued to discuss some degree grounded topological descriptors in association with their corresponding entropy measures. We extend this computation to the quantitative and pictorial comparisons which could be beneficial in the structure amendment for effective implementation.
\end{abstract}

\section{Introduction}

Chemical graph theory is a fascinating branch of mathematics and is the combination of chemistry and graph theory. In molecular graph, molecules are designed mathematically. In a molecular graph, the vertices and edges portrayed the atoms and the bonds correspondingly. Various approaches are employed on molecular graphs to acquire various structure properties of corresponding compounds. A numerical worth treated theoretically from the atomic graph is named as topological index. It is connected with synthetic constitution exhibiting for association of substance structure with miscellaneous physical and natural exercises and concoction properties. The degreebased topological indices collaborate actively in the exploitation of different fields specifically in pharmaceutical and commercial chemistry. In [1], Randić index is originated, while $A B C$ index, GA index, first Zagreb index, and second Zagreb were discussed in [2, 3]. In [4], hyper-Zagreb index was introduced. In [5], forgotten index was initiated.
Furtula [6] established the concept of AZI index. Balaban $[7,8]$ laid the foundation of Balaban index. The redefined first, second, and third Zagreb indices were investigated in [9]. The forth atom bond connectivity index was presented in [10]. The fifth geometric arithmetic index is declared in [11]. The specific topological indices of some graphs are discussed in $[12,13]$. An ordered pair $\left(M_{V}, M_{E}\right)$, composed of a nonempty set $M_{V}$ of vertices and $M_{E}$ as a set of edges, is called a graph. If any two vertices $m$ and $n$ are attached with each other, they make an edge $e$. The number of edges adjacent with the vertex $m$ is characterized as $\tau(m)$.

Entropy has been a comprehensive and transcendental approach in diverse areas of expertise diverging from logic and biology to physics and engineering. Entropy connects the conception of randomness and uncertainty with physical conditions which are constructed as transformation channels of information. This study represents the special case when these channels are the detectors investigating the response of a system. Formally, entropy came into consideration after the invention of the heat engine, as a 
consequence of pioneering investigation towards explaining thermodynamical techniques and expanding the efficacy of such machines $[14,15]$. This investigation reveals that the entropy of a system or machine cannot decrease over time. In 1948, Claude Shannon [16] introduces a measure of uncertainty as entropy. It can be explicated as the rate of production of new information manufactured by the system [17-19]. As stated by Shannon, uncertainty and information are two sides of the coin: a reduction in uncertainty is the same as the reception of a certain amount of information. Therefore, as the value of the entropy about a system is greater, the uncertainty about its response is also increased. In literature, many graph entropies are estimated; see [20-23].

In 2014, Chen et al. [24] introduced the definition of the entropy of edge weighted graph $M=\left(M_{V} ; M_{E} ; \psi(m n)\right)$. The entropy formula is represented in

$$
\begin{aligned}
E_{\psi}(M) & =-\sum_{i=1}^{m} F_{i} \frac{\psi\left(m_{i} n_{i}\right)}{I} \log \frac{\psi\left(m_{i} n_{i}\right)}{I} \\
& =\log (I)-\frac{1}{I} \sum_{i=1}^{m} F_{i} \psi\left(m_{i} n_{i}\right) \log \psi\left(m_{i} n_{i}\right) .
\end{aligned}
$$

\section{Crystallographic Structure of $\mathbf{S i}_{2} \mathbf{C}_{3}-I[m, n]$}

The most consistent framework of 2D silicon carbon unicellular compound is anticipated which is grounded on the (PSO) particle swarm optimization approach. It is rich in carbon. In the last decades, silicon carbon was considered to be the hardest material across the world. Due to its Mohs hardness rating, it resembles diamond. Silicon is of low cost and is nontoxic semiconductor. Numerous studies have been done in the refinement, magnification, and device assembling [25]. It is employed for all advanced electronic gadgets. The silicon carbide framework may seem like the honeycomb framework of graphene. Despite that, large scale search proposes that the framework of this compound is absolutely distinct compared to that of graphene. For silicon carbide $I\left(\mathrm{Si}_{2} \mathrm{C}_{3}\right)$ layer, the minimum energy of $\mathrm{Si}_{2} \mathrm{C}_{3}-I$ demonstrates a planar framework comprised of polygonal rings, where two pentagonal and four heptagonal rings encircling each hexagonal ring. See Figure 1 [26]. Every hexagonal ring carries out three $\mathrm{Si}$ and three $\mathrm{C}$ atoms in which $\mathrm{Si}$ and $\mathrm{C}$ atoms are positioned by turn on the vertices. Pentagonal rings are of two kinds in which one is comprised of two Si and three $\mathrm{C}$ atoms. Also, three Si and four $\mathrm{C}$ atoms establish the heptagonal rings. It is to be noted that there are no $\mathrm{Si}-\mathrm{Si}$ bonds in $\mathrm{Si}_{2} \mathrm{C}_{3}-I$ sheet. Here $m$ denotes the total associated unit cells in each row while $n$ shows the total sum of associated rows with $m$ number of cells.

Figure 2 illustrates the way in which cells are associated in a row and the way of association of rows with each other. Also, $\left|V\left(\mathrm{Si}_{2} \mathrm{C}_{3}-I\right)\right|=10 m n$ and $\left|E\left(\mathrm{Si}_{2} \mathrm{C}_{3}-I\right)\right|=3(5 m n$ $-n)-2 m$.

2.1. Formation of $\mathrm{Si}_{2} \mathrm{C}_{3}-I[m, n]$ Formulas. Here, we join one unit cell with another unit cell and then continue this process horizontally till $n$ unit cells. Similar technique will be applied for vertical direction. As a result, we will obtain the $\mathrm{Si}_{2} \mathrm{C}_{3}-I[m, n]$ sheet; see Figure 1 . The vertex and edge partition are depicted in Tables 1 and 2, respectively.

Table 3 shows the edge partition of the chemical graph $\mathrm{Si}_{2} \mathrm{C}_{3}-I[m, n]$ based on degree sum of end vertices.

2.2. Computation of Entropies for Crystallographic Structure of $\mathrm{Si}_{2} \mathrm{C}_{3}-I[m, n]$. This portion deals with the computation of topological indices and their corresponding graph entropies for the crystallographic structure of $\mathrm{Si}_{2} \mathrm{C}_{3}-I[m, n]$.

2.2.1. The Randic Index and Randić Entropy for $\mathrm{Si}_{2} \mathrm{C}_{3}-I[m, n]$. For $\alpha=1$, the Randić index and Randić entropy by using Table 2 are

$$
\begin{aligned}
R_{1}\left(\mathrm{Si}_{2} \mathrm{C}_{3}-I\right)= & 135 m n-41 m-61 n+14, \\
E_{R_{1}}\left(\mathrm{Si}_{2} \mathrm{C}_{3}-I\right)= & \log [135 m n-41 m-61 n+14] \\
& -\frac{\log (2)^{(2)}}{135 m n-41 m-61 n+14} \\
& -\frac{\log (3)^{(3)}}{135 m n-41 m-61 n+14} \\
& -\frac{\log \left[(m+2 n) \times(4)^{(4)}\right]}{135 m n-41 m-61 n+14} \\
& -\frac{\log \left[(6 m+8 n-9) \times(6)^{(6)}\right]}{135 m n-41 m-61 n+14} \\
& -\frac{\log \left[(15 m n-9 m-13 n+7) \times(9)^{(9)}\right]}{135 m n-41 m-61 n+14} .
\end{aligned}
$$

For $\alpha=-1$, by using Table 2 , the Randić index and Randić entropy are

$$
\begin{aligned}
R_{-1}\left(\mathrm{Si}_{2} C_{3}-I\right)= & \frac{5 m n}{3}+\frac{m}{4}+\frac{7 n}{18}+\frac{1}{9} \\
E_{R_{-1}}\left(\mathrm{Si}_{2} C_{3}-I\right)= & \log \left(\frac{5}{3} m n+\frac{1}{4} 4+\frac{7}{18} n+\frac{1}{9}\right) \\
& -\frac{\left.\log \left[(2)^{(-1 / 2)}\right]\right]}{((5 / 3) m n+(1 / 4) 4+(7 / 18) n+(1 / 9))} \\
& -\frac{\left.\log \left[(3)^{(-1 / 3)}\right]\right]}{((5 / 3) m n+(1 / 4) 4+(7 / 18) n+(1 / 9))} \\
& -\frac{\log \left[(m+2 n) \times(4)^{(-1 / 4)}\right]}{((5 / 3) m n+(1 / 4) 4+(7 / 18) n+(1 / 9))} \\
& -\frac{\log \left[(6 m+8 n-9) \times(6)^{(-1 / 6)}\right]}{((5 / 3) m n+(1 / 4) 4+(7 / 18) n+(1 / 9))} \\
& -\frac{\log \left[(15 m n-9 m-13 n+7) \times(9)^{(-1 / 9)}\right]}{((5 / 3) m n+(1 / 4) 4+(7 / 18) n+(1 / 9))} .
\end{aligned}
$$




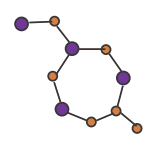

(a)

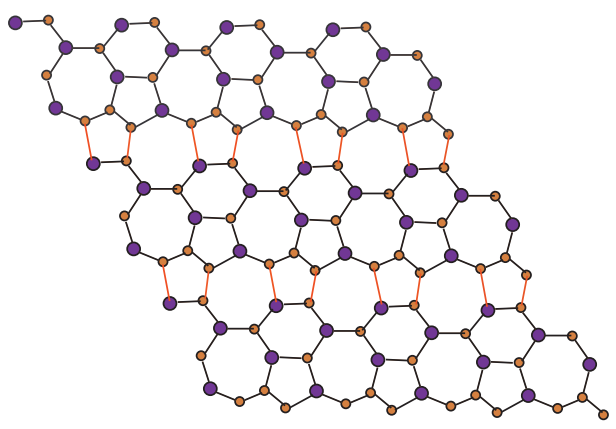

(b)

Figure 1: (a) Unit cell of $\mathrm{Si}_{2} \mathrm{C}_{3}-I[m, n]$; (b) $\mathrm{Si}_{2} \mathrm{C}_{3}-I[4,3]$.

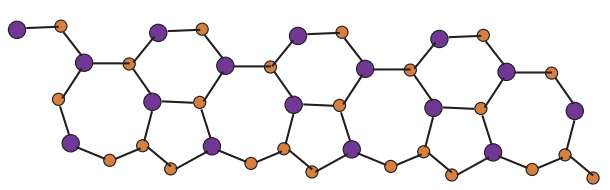

(a)

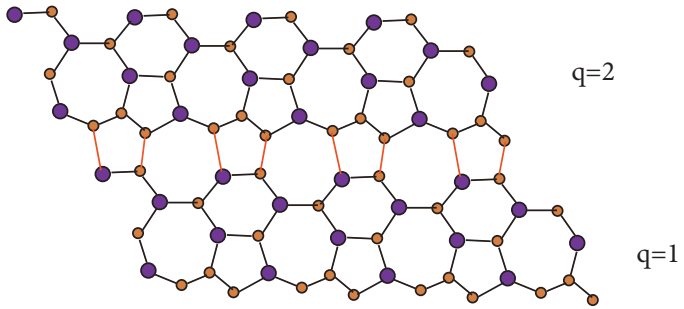

(b)

Figure 2: (a) $\mathrm{Si}_{2} \mathrm{C}_{3}-I[4,1]$, one row with $m=4$ and $n=1$; (b) $\mathrm{Si}_{2} \mathrm{C}_{3}-I[4,2]$.

TABLe 1: Vertex partition of $\mathrm{Si}_{2} \mathrm{C}_{3}-I[m, n]$.

\begin{tabular}{lcc}
\hline$\tau(m)$ & Frequency & Kind of vertices \\
\hline 1 & 2 & $M_{V_{1}}$ \\
2 & $4 m+6 n-4$ & $M_{V_{2}}$ \\
3 & $10 m n-4 m-6 n+2$ & $M_{V_{2}}$ \\
\hline
\end{tabular}

TABLE 2: Edge partition of $\mathrm{Si}_{2} \mathrm{C}_{3}-I[m, n]$.

\begin{tabular}{lcc}
\hline$(\tau(m), \tau(n))$ & Frequency & Set of edges \\
\hline$(2,1)$ & 1 & $M_{E_{1}}$ \\
$(3,1)$ & 1 & $M_{E_{2}}$ \\
$(2,2)$ & $m+2 n$ & $M_{E_{3}}$ \\
$(3,2)$ & $6 m+8 n-9$ & $M_{E_{4}}$ \\
$(3,3)$ & $15 m n-9 m-13 n+7$ & $M_{E_{5}}$ \\
\hline
\end{tabular}

TABLE 3: Edge partition of $\mathrm{Si}_{2} \mathrm{C}_{3}-I[m, n]$ with $m, n \geq 2$.

\begin{tabular}{lc}
\hline$\left(S_{m}, S_{n}\right)$ & Frequency \\
\hline$(4,2)$ & 1 \\
$(5,3)$ & 1 \\
$(5,5)$ & $m+2 n$ \\
$(5,5)$ & 1 \\
$(7,4)$ & 1 \\
$(7,5)$ & $2+2 n$ \\
$(5,6)$ & 1 \\
$(7,6)$ & $-7+2(2 m+n)$ \\
$(8,6)$ & $2(-1+n)$ \\
$(8,5)$ & $-5+2(m+n)$ \\
$(8,7)$ & 1 \\
$(8,8)$ & $-4+2 n+m$ \\
$(9,7)$ & $-3+2(m+n)$ \\
$(9,8)$ & $-7+2(m+2 n)$ \\
$(9,9)$ & $15 m n-14 m-21 n+20$ \\
\hline
\end{tabular}


For $\alpha=1 / 2$, by using Table 2 , the Randić index and Randić entropy are

$$
\begin{aligned}
& R_{1 / 2}\left(\mathrm{Si}_{2} \mathrm{C}_{3}-I\right)=45 m n+(6 \sqrt{6}-27) m+(8 \sqrt{6}-35) n+\sqrt{2}+\sqrt{3}-9 \sqrt{6}+21, \\
& E_{R_{1 / 2}}\left(\mathrm{Si}_{2} \mathrm{C}_{3}-I\right)=\log (45 m n+(6 \sqrt{6}-27) m+(8 \sqrt{6}-35) n+\sqrt{2}+\sqrt{3}-9 \sqrt{6}+21) \\
& \log \left[1 \times(2)^{((1 / 2) \cdot \sqrt{2})}\right] \\
& -\frac{(45 m n+(6 \sqrt{6}-27) m+(8 \sqrt{6}-35) n+\sqrt{2}+\sqrt{3}-9 \sqrt{6}+21)}{(-2)} \\
& -\frac{\log \left[1 \times(3)^{((1 / 2) \cdot \sqrt{3})}\right]}{(45 m n+(6 \sqrt{6}-27) m+(8 \sqrt{6}-35) n+\sqrt{2}+\sqrt{3}-9 \sqrt{6}+21)} \\
& -\frac{\log \left[(m+2 n) \times(4)^{((1 / 2) \cdot \sqrt{4})}\right]}{(45 m n+(6 \sqrt{6}-27) m+(8 \sqrt{6}-35) n+\sqrt{2}+\sqrt{3}-9 \sqrt{6}+21)} \\
& -\frac{\log \left[(6 m+8 n-9) \times(6)^{((1 / 2) \cdot \sqrt{6})}\right]}{(45 m n+(6 \sqrt{6}-27) m+(8 \sqrt{6}-35) n+\sqrt{2}+\sqrt{3}-9 \sqrt{6}+21)} \\
& -\frac{\log \left[(15 m n-9 m-13 n+7) \times(9)^{((1 / 2) \cdot \sqrt{9})}\right]}{(45 m n+(6 \sqrt{6}-27) m+(8 \sqrt{6}-35) n+\sqrt{2}+\sqrt{3}-9 \sqrt{6}+21)} .
\end{aligned}
$$

For $\alpha=-1 / 2$, by using and Table 2 , the Randić index and Randić entropy are

$$
\begin{aligned}
& R_{-1 / 2}\left(\mathrm{Si}_{2} \mathrm{C}_{3}-I\right)=5 m n+\left(\sqrt{6}-\frac{5}{2}\right) m+\left(\frac{8}{\sqrt{6}}-\frac{10}{3}\right) n+\frac{1}{\sqrt{2}}+\frac{1}{\sqrt{3}}-\frac{9}{\sqrt{6}}+\frac{7}{3}, \\
& E_{R_{-1 / 2}}\left(\mathrm{Si}_{2} \mathrm{C}_{3}-I\right)=\log \left(5 m n+\left(\sqrt{6}-\frac{5}{2}\right) m+\left(\frac{8}{\sqrt{6}}-\frac{10}{3}\right) n+\frac{1}{\sqrt{2}}+\frac{1}{\sqrt{3}}-\frac{9}{\sqrt{6}}+\frac{7}{3}\right) \\
& \log \left[1 \times(2)^{((-1 / 2) \cdot(1 / \sqrt{2}))}\right] \\
& (5 m n+(\sqrt{6}-(5 / 2)) m+((8 / \sqrt{6})-(10 / 3)) n+(1 / \sqrt{2})+(1 / \sqrt{3})-(9 / \sqrt{6})+(7 / 3)) \\
& -\frac{\log \left[1 \times(3)^{((-1 / 2) \cdot(1 / \sqrt{3}))}\right]}{(5 m n+(\sqrt{6}-(5 / 2)) m+((8 / \sqrt{6})-(10 / 3)) n+(1 / \sqrt{2})+(1 / \sqrt{3})-(9 / \sqrt{6})+(7 / 3))} \\
& \log \left[(m+2 n) \times(4)^{((-1 / 2) \cdot(1 / \sqrt{4}))}\right] \\
& -\frac{(5 m n+(\sqrt{6}-(5 / 2)) m+((8 / \sqrt{6})-(10 / 3)) n+(1 / \sqrt{2})+(1 / \sqrt{3})-(9 / \sqrt{6})+(7 / 3))}{(5)} \\
& \log \left[(6 m+8 n-9) \times(6)^{((-1 / 2) \cdot(1 / \sqrt{6}))}\right] \\
& -\overline{(5 m n+(\sqrt{6}-(5 / 2)) m+((8 / \sqrt{6})-(10 / 3)) n+(1 / \sqrt{2})+(1 / \sqrt{3})-(9 / \sqrt{6})+(7 / 3))} \\
& \log \left[(15 m n-9 m-13 n+7) \times(9)^{((-1 / 2) \cdot(1 / \sqrt{9}))}\right] \\
& (5 m n+(\sqrt{6}-(5 / 2)) m+((8 / \sqrt{6})-(10 / 3)) n+(1 / \sqrt{2})+(1 / \sqrt{3})-(9 / \sqrt{6})+(7 / 3))
\end{aligned}
$$


2.2.2. The $A B C$ Index and $A B C$ Entropy of $S i_{2} C_{3}-I[m, n] . \quad$ By using Table 2, the $A B C$ index and $A B C$ entropy are

$$
\begin{aligned}
A B C\left(\mathrm{Si}_{2} \mathrm{C}_{3}-I\right)= & 10 m n+\left(\frac{6}{\sqrt{2}}+\frac{1}{\sqrt{2}}-6\right) m+\left(\frac{2}{\sqrt{2}}+\frac{8}{\sqrt{2}}-\frac{26}{3}\right) n-\frac{8}{\sqrt{2}}+\sqrt{\frac{2}{3}}+\frac{14}{3} \\
E_{A B C}\left(\mathrm{Si}_{2} \mathrm{C}_{3}-I\right)= & \log \left(10 m n+\left(\frac{6}{\sqrt{2}}+\frac{1}{\sqrt{2}}-6\right) m+\left(\frac{2}{\sqrt{2}}+\frac{8}{\sqrt{2}}-\frac{26}{3}\right) n-\frac{8}{\sqrt{2}}+\sqrt{\frac{2}{3}}+\frac{14}{3}\right) \\
& -\frac{\log \left[1 \times(1 / \sqrt{2})^{(1 / \sqrt{2})}\right]}{(10 m n+((6 / \sqrt{2})+(1 / \sqrt{2})-6) m+((2 / \sqrt{2})+(8 / \sqrt{2})-(26 / 3)) n-(8 / \sqrt{2})+\sqrt{(2 / 3)}+(14 / 3)} \\
& -\frac{\log [1 \times(\sqrt{(2 / 3)})(\sqrt{(2 / 3)})]}{(10 m n+((6 / \sqrt{2})+(1 / \sqrt{2})-6) m+((2 / \sqrt{2})+(8 / \sqrt{2})-(26 / 3)) n-(8 / \sqrt{2})+\sqrt{(2 / 3)}+(14 / 3)} \\
& -\frac{\log \left[(m+2 n) \times(1 / \sqrt{2})^{(1 / \sqrt{2})}\right]}{(10 m n+((6 / \sqrt{2})+(1 / \sqrt{2})-6) m+((2 / \sqrt{2})+(8 / \sqrt{2})-(26 / 3)) n-(8 / \sqrt{2})+\sqrt{(2 / 3)}+(14 / 3)} \\
& -\frac{\log [(6 m+8 n-9) \times(1 / \sqrt{2})(1 / \sqrt{2})]}{\log [(15 m n-9 m-13 n+7) \times(2 / 3)(2 / 3)]}
\end{aligned}
$$

2.2.3. The GA Index and GA Entropy of $S i_{2} C_{3}-I[m, n]$. By using Table 2, the GA index and GA entropy are

$$
\begin{aligned}
\mathrm{GA}\left(\mathrm{Si}_{2} \mathrm{C}_{3}-I\right)= & 15 m n+\left(\frac{12 \sqrt{6}}{5}-8\right) m+\left(\frac{16 \sqrt{6}}{5}-11\right) n+\frac{2 \sqrt{2}}{3}+\frac{\sqrt{3}}{2}-\frac{18 \sqrt{6}}{5}+7 \\
E_{\mathrm{GA}}\left(\mathrm{Si}_{2} \mathrm{C}_{3}-I\right)= & \log \left[15 m n+\left(\frac{12 \sqrt{6}}{5}-8\right) m+\left(\frac{16 \sqrt{6}}{5}-11\right) n+\frac{2 \sqrt{2}}{3}+\frac{\sqrt{3}}{2}-\frac{18 \sqrt{6}}{5}+7\right] \\
& -\frac{\log \left[1 \times[(2 \sqrt{2} / 3)]^{[(2 \sqrt{2} / 3)]}\right]}{(15 m n+((12 \sqrt{6} / 5)-8) m+((16 \sqrt{6} / 5)-11) n+(2 \sqrt{2} / 3)+(\sqrt{3} / 2)-(18 \sqrt{6} / 5)+7)} \\
& -\frac{\log \left[1 \times[(\sqrt{3} / 2)]^{[(\sqrt{3} / 2)]}\right]}{(15 m n+((12 \sqrt{6} / 5)-8) m+((16 \sqrt{6} / 5)-11) n+(2 \sqrt{2} / 3)+(\sqrt{3} / 2)-(18 \sqrt{6} / 5)+7)} \\
& -\frac{\log [1 \times(m+2 n)]}{(15 m n+((12 \sqrt{6} / 5)-8) m+((16 \sqrt{6} / 5)-11) n+(2 \sqrt{2} / 3)+(\sqrt{3} / 2)-(18 \sqrt{6} / 5)+7)} \\
& -\frac{\log [(15 m n-9 m-13 n+7) \times 1]}{(15 m n+((12 \sqrt{6} / 5)-8) m+((16 \sqrt{6} / 5)-11) n+(2 \sqrt{2} / 3)+(\sqrt{3} / 2)-(18 \sqrt{6} / 5)+7)}
\end{aligned}
$$


2.2.4. The First Zagreb Index and First Zagreb Entropy of $\mathrm{Si}_{2} \mathrm{C}_{3}-I[m, n]$. By using Table 2, the first Zagreb index and entropy are

$$
\begin{aligned}
M_{1}\left(\mathrm{Si}_{2} \mathrm{C}_{3}-I\right)= & 90 m n-20 m-30 n+4 \\
E_{M_{1}}\left(\mathrm{Si}_{2} \mathrm{C}_{3}-I\right)= & \log (90 m n-20 m-30 n+4) \\
& -\frac{\log [(1) \times(27)]}{(90 m n-20 m-30 n+4)} \\
& -\frac{\log [(1) \times(256)]}{(90 m n-20 m-30 n+4)} \\
& -\frac{\log [(m+2 n) \times(3125)]}{(90 m n-20 m-30 n+4)} \\
& -\frac{\log [(15 m n-9 m-13 n+7) \times(46656)]}{(90 m n-20 m-30 n+4)} .
\end{aligned}
$$

2.2.5. The Second Zagreb Index and Second Zagreb Entropy of $\mathrm{Si}_{2} \mathrm{C}_{3}-I[m, n]$. By using Table 2, the second Zagreb index and second Zagreb entropy are

$$
\begin{aligned}
M_{2}\left(\mathrm{Si}_{2} \mathrm{C}_{3}-I\right)= & 135 m n-9 m-61 n+14, \\
E_{M_{2}}\left(\mathrm{Si}_{2} \mathrm{C}_{3}-I\right)= & \log (135 m n-9 m-61 n+14) \\
& -\frac{\log [(1) \times(4)]}{(135 m n-9 m-61 n+14)} \\
& -\frac{\log [(1) \times(27)]}{(135 m n-9 m-61 n+14)} \\
& -\frac{\log [[(m+2 n) \times(256)]]}{(135 m n-9 m-61 n+14)} \\
& -\frac{\log [(6 m+8 n-9) \times(46656)]}{(135 m n-9 m-61 n+14)} \\
& -\frac{\log \left[(15 m n-9 m-13 n+7) \times\left(9^{9}\right)\right]}{(135 m n-9 m-61 n+14)} .
\end{aligned}
$$

2.2.6. The Hyper-Zagreb Index and Hyper-Zagreb Entropy of $\mathrm{Si}_{2} \mathrm{C}_{3}-I[m, n]$. By using Table 2, the hyper-Zagreb index and hyper-Zagreb entropy are

$$
\begin{aligned}
\mathrm{HM}\left(\mathrm{Si}_{2} \mathrm{C}_{3}-I\right)= & 540 m n-158 m-236 n+412, \\
E_{\mathrm{HM}}\left(\mathrm{Si}_{2} \mathrm{C}_{3}-I\right)= & \log (540 m n-158 m-236 n+412) \\
& -\frac{\log \left[(9)^{(9)}\right]}{(540 m n-158 m-236 n+412)} \\
& -\frac{\log \left[(16)^{(16)}\right]}{(540 m n-158 m-236 n+412)} \\
& -\frac{\log \left[(m+2 n) \times(16)^{(16)}\right]}{(540 m n-158 m-236 n+412)} \\
& -\frac{\log \left[(6 m+8 n-9) \times(25)^{(25)}\right]}{(540 m n-158 m-236 n+412)} \\
& -\frac{\log \left[(15 m n-9 m-13 n+7) \times(36)^{(36)}\right]}{(540 m n-158 m-236 n+412)} .
\end{aligned}
$$

2.2.7. The Forgotten Index and Forgotten Entropy for $\mathrm{Si}_{2} \mathrm{C}_{3}-I[m, n]$. By using Table 2, the forgotten index and forgotten entropy are

$$
\begin{aligned}
F\left(\mathrm{Si}_{2} \mathrm{C}_{3}-I\right)= & 270 m n-76 m-114 n+24, \\
E_{F}\left(\mathrm{Si}_{2} \mathrm{C}_{3}-I\right)= & \log (270 m n-76 m-114 n+24) \\
& -\frac{\log \left[(5)^{(5)}\right]}{(270 m n-76 m-114 n+24)} \\
& -\frac{\log \left[(10)^{(10)}\right]}{(270 m n-76 m-114 n+24)} \\
& -\frac{\log \left[(m+2 n) \times(8)^{(8)}\right]}{(270 m n-76 m-114 n+24)} \\
& -\frac{\log \left[(6 m+8 n-9) \times(13)^{(13)}\right]}{(270 m n-76 m-114 n+24)} \\
& -\frac{\log \left[(15 m n-9 m-13 n+7) \times(18)^{(18)}\right]}{(270 m n-76 m-114 n+24)} .
\end{aligned}
$$

2.2.8. The Augmented Zagreb Index and Augmented Zagreb Entropy of $\mathrm{Si}_{2} \mathrm{C}_{3}-I[m, n]$. By using Table 2, the AZI index and AZI entropy are $\mathrm{AZI}\left(\mathrm{Si}_{2} \mathrm{C}_{3}-I\right)=(10935 / 64) m n-$ $(3481 / 64) m-(4357 / 64) n+(1223 / 64)$

$$
E_{\mathrm{AZI}}\left(\mathrm{Si}_{2} \mathrm{C}_{3}-I\right)=\log \left(\frac{10935}{64} m n-\frac{3481}{64} m-\frac{4357}{64} n+\frac{1223}{64}\right)
$$




$$
\begin{aligned}
& -\frac{\log \left[(8)^{(8)}\right]}{((10935 / 64) m n-(3481 / 64) m-(4357 / 64) n+(1223 / 64))}-\frac{\log \left[(27 / 8)^{(27 / 8)}\right]}{((10935 / 64) m n-(3481 / 64) m-(4357 / 64) n+(1223 / 64))} \\
& -\frac{\log \left[(m+2 n) \times(8)^{(8)}\right]}{((10935 / 64) m n-(3481 / 64) m-(4357 / 64) n+(1223 / 64))}-\frac{\log \left[(15 m n-9 m-13 n+7) \times\left[\left(9^{3} / 4^{3}\right)\right]^{\left[\left(9^{3} / 4^{3}\right)\right]}\right]}{((10935 / 64) m n-(3481 / 64) m-(4357 / 64) n+(1223 / 64))}
\end{aligned}
$$

2.2.9. The Balaban Index and Balaban Entropy for $\mathrm{Si}_{2} \mathrm{C}_{3}-I[m, n]$. By using Table 2, the Balaban index and Balaban entropy are

$$
\begin{aligned}
J\left(\mathrm{Si}_{2} \mathrm{C}_{3}-I\right)= & \frac{15 m n-2 m-3 n}{5 m n-2 m-3 n+2}\left[\frac{1}{\sqrt{2}}+\frac{1}{\sqrt{3}}+\frac{1}{2}(m+2 n)+\frac{1}{\sqrt{6}}(6 m+8 n-9)+\frac{1}{3}(15 m n-9 m-13 n+7)\right] \\
\mathrm{ENT}_{J}\left(\mathrm{Si}_{2} \mathrm{C}_{3}-I\right)= & \log (J(G))-\frac{[q /(\sqrt{2}(q-p+2))] \log [q /(\sqrt{2}(q-p+2))]}{(J(G))}-\frac{[q /(\sqrt{3}(q-p+2))] \log [q /(\sqrt{3}(q-p+2))]}{(J(G))} \\
& -\frac{[q /(2(q-p+2))] \log [(m+2 n) \times[q /(2(q-p+2))]]}{(J(G))} \\
& -\frac{[q /(\sqrt{6}(q-p+2))] \log [q(6 m+8 n-9) /(\sqrt{6}(q-p+2))]}{(J(G))} \\
& -\frac{[q / 3(q-p+2)] \log [(15 m n-9 m-13 n+7) \times(q / 3(q-p+2))]}{(J(G))} .
\end{aligned}
$$

2.2.10. The Redefined First Zagreb Index and Redefined First Zagreb Entropy for $\mathrm{Si}_{2} \mathrm{C}_{3}-I[m, n]$. By using Table 2, the redefined first Zagreb index and redefined first Zagreb entropy are $\operatorname{ReZG}_{1}\left(\mathrm{Si}_{2} \mathrm{C}_{3}-I\right)=10 m n$ and

$$
\begin{aligned}
E_{\mathrm{ReZG}_{1}}\left(\mathrm{Si}_{2} \mathrm{C}_{3}-I\right)= & \log (10 m n)-\frac{\log \left[(3 / 2)^{(3 / 2)}\right]}{(10 m n)}-\frac{\log \left[(4 / 3)^{(4 / 3)}\right]}{(10 m n)}-\frac{\log [m+2 n]}{(10 m n)} \\
& -\frac{\log \left[(6 m+8 n-9) \times(5 / 6)^{(5 / 6)}\right]}{(10 m n)}-\frac{\log \left[(15 m n-9 m-13 n+7) \times(2 / 3)^{(2 / 3)}\right]}{(10 m n)} .
\end{aligned}
$$

2.2.11. The Redefined Second Zagreb Index and Redefined Second Zagreb Entropy for $\mathrm{Si}_{2} \mathrm{C}_{3}-I[m, n]$. By using Table 2, the redefined second Zagreb index and redefined second
Zagreb entropy are $\operatorname{ReZG}_{2}\left(\mathrm{Si}_{2} \mathrm{C}_{3}-I\right)=(45 / 2) m n-$ $(53 / 10) m-(79 / 10) n+(67 / 60)$ and

$$
\begin{aligned}
E_{\mathrm{ReZG}_{2}}\left(\mathrm{Si}_{2} \mathrm{C}_{3}-I\right)= & \log \left(\frac{45}{2} m n-\frac{53}{10} m-\frac{79}{10} n+\frac{67}{60}\right)-\frac{\log \left[(2 / 3)^{(2 / 3)}\right]}{((45 / 2) m n-(53 / 10) m-(79 / 10) n+(67 / 60))} \\
& -\frac{\log \left[(3 / 4)^{(3 / 4)}\right]}{((45 / 2) m n-(53 / 10) m-(79 / 10) n+(67 / 60))}
\end{aligned}
$$




$$
\begin{aligned}
& -\frac{\log [m+2 n]}{((45 / 2) m n-(53 / 10) m-(79 / 10) n+(67 / 60))}-\frac{\log \left[(6 / 5)^{(6 / 5)}(6 m+8 n-9)\right]}{((45 / 2) m n-(53 / 10) m-(79 / 10) n+(67 / 60))} \\
& -\frac{\log \left[(3 / 2)^{(3 / 2)}(15 m n-9 m-13 n+7)\right]}{((45 / 2) m n-(53 / 10) m-(79 / 10) n+(67 / 60))} .
\end{aligned}
$$

2.2.12. The Redefined Third Zagreb Index and Redefined Third Zagreb Entropy for $\mathrm{Si}_{2} \mathrm{C}_{3}-I[m, n]$. By using Table 2, the redefined third Zagreb index and redefined third Zagreb entropy are $\operatorname{ReZG}_{3}\left(\mathrm{Si}_{2} \mathrm{C}_{3}-I\right)=810 m n-290 m-430 n$ +126 and

$$
\begin{aligned}
E_{\mathrm{ReZG}_{3}}\left(\mathrm{Si}_{2} \mathrm{C}_{3}-I\right)= & \log (810 m n-290 m-430 n+126)-\frac{\log \left[\left[(1) \times(6)^{(6)}\right]\right]}{(810 m n-290 m-430 n+126)} \\
& -\frac{\log \left[(1) \times(12)^{(12)}\right]}{(810 m n-290 m-430 n+126)}-\frac{\log \left[\left[(m+2 n) \times(16)^{(16)}\right]\right]}{(810 m n-290 m-430 n+126)} \\
& -\frac{\log \left[(6 m+8 n-9) \times(30)^{(30)}\right]}{(810 m n-290 m-430 n+126)}-\frac{\log \left[(15 m n-9 m-13 n+7) \times(54)^{(54)}\right]}{(810 m n-290 m-430 n+126)} .
\end{aligned}
$$

2.2.13. The Fourth $A B C$ Index and Fourth $A B C$ Entropy of $S_{2} C_{3}-I[m, n]$. By using Table 3 , the fourth $A B C$ index and fourth $A B C$ entropy are

$$
\begin{aligned}
& A B C_{4}\left(\mathrm{Si}_{2} \mathrm{C}_{3}-I\right)=\frac{20}{3} m n+\left[\frac{\sqrt{8}}{5}+4 \times \sqrt{\left(\frac{11}{42}\right)}+2 \times \sqrt{\left(\frac{11}{40}\right)}+2 \frac{\sqrt{2}}{3}+\frac{\sqrt{14}}{8}+\sqrt{\left(\frac{5}{6}\right)}-\frac{56}{9}\right] m \\
& +\left[4 \frac{\sqrt{2}}{5}+2 \sqrt{\left(\frac{2}{7}\right)}+2 \sqrt{\left(\frac{11}{42}\right)}+\sqrt{\left(\frac{11}{10}\right)}+\frac{7}{2 \sqrt{2}}+\frac{2 \sqrt{2}}{3}+\frac{\sqrt{14}}{4}+2 \sqrt{\left(\frac{5}{6}\right)}-\frac{25}{3}\right] n \\
& +\frac{1}{\sqrt{2}}+\sqrt{\left(\frac{2}{5}\right)}+\frac{\sqrt{8}}{5}+\frac{3}{2 \sqrt{7}}+2 \sqrt{\left(\frac{2}{7}\right)}+\sqrt{\left(\frac{3}{10}\right)}-7 \sqrt{\left(\frac{11}{42}\right)}-5 \sqrt{\left(\frac{11}{40}\right)} \\
& +\sqrt{\left(\frac{13}{56}\right)}-\sqrt{\left(\frac{7}{2}\right)}-\sqrt{2}-\frac{7}{2} \sqrt{\left(\frac{13}{56}\right)}+\frac{71}{9} \\
& \mathrm{ENT}_{A B C_{4}}(G)=\log \left(A B C_{4}\right)-\frac{1}{\left(A B C_{4}\right)} \log \left[\left(\frac{1}{\sqrt{2}}\right)^{(1 / \sqrt{2})}\right]-\frac{1}{\left(A B C_{4}\right)} \log \left[\left(\sqrt{\frac{2}{5}}\right)^{(\sqrt{(2 / 5)})}\right]-\frac{1}{\left(A B C_{4}\right)} \log \left[\left(\frac{3}{2 \sqrt{7}}\right)^{(3 / 2 \sqrt{7})}\right] \\
& -\frac{1}{\left(A B C_{4}\right)} \log \left[(m+2 n+1) \times\left(\frac{\sqrt{8}}{5}\right)^{(\sqrt{8} / 5)}\right]-\frac{1}{\left(A B C_{4}\right)} \log \left[(2 n+2) \times\left(\sqrt{\frac{2}{7}}\right)^{(\sqrt{(2 / 7)})}\right] \\
& -\frac{1}{\left(A B C_{4}\right)} \log \left[\left(\sqrt{\frac{3}{10}}\right)^{(\sqrt{(3 / 10)})}\right]-\frac{1}{\left(A B C_{4}\right)} \log \left[(4 m+2 n-7) \times\left(\sqrt{\frac{11}{42}}\right)^{(\sqrt{(11 / 42)})}\right] \\
& -\frac{1}{\left(A B C_{4}\right)} \log \left[(2 n-2) \times\left(\frac{1}{2}\right)^{(1 / 2)}\right]-\frac{1}{\left(A B C_{4}\right)} \log \left[(2 m+2 n-5) \times\left(\sqrt{\frac{11}{40}}\right)^{(\sqrt{(11 / 40)})}\right]
\end{aligned}
$$




$$
\begin{aligned}
& -\frac{1}{\left(A B C_{4}\right)} \log \left[\left(\sqrt{\frac{13}{56}}\right)^{(\sqrt{(13 / 56)})}\right]-\frac{1}{\left(A B C_{4}\right)} \log \left[(m+2 n-4) \times\left(\frac{\sqrt{14}}{8}\right)^{(\sqrt{14} / 8)}\right] \\
& -\frac{1}{\left(A B C_{4}\right)} \log \left[(2 m+2 n-3) \times\left(\frac{\sqrt{2}}{3}\right)^{(\sqrt{2} / 3)}\right]-\frac{1}{\left(A B C_{4}\right)} \log \left[(2 m+4 n-7) \times\left(\frac{\sqrt{5}}{2 \sqrt{6}}\right)^{(\sqrt{5} / 2 \sqrt{6})}\right] \\
& -\frac{1}{\left(A B C_{4}\right)} \log \left[(15 m n-14 m-21 n+20) \times\left(\frac{4}{9}\right)^{(4 / 9)}\right] .
\end{aligned}
$$

2.2.14. The Fifth GA Index and Fifth GA Entropy of $\mathrm{Si}_{2} \mathrm{C}_{3}-I[m, n]$. By using Table 3 , the fifth GA index and fifth GA entropy are

$$
\begin{aligned}
& \mathrm{GA}_{5}(G)=15 m n+\left(\frac{8 \sqrt{42}}{13}+\frac{8 \sqrt{10}}{13}+\frac{3 \sqrt{7}}{4}+\frac{24 \sqrt{2}}{17}-12\right) m \\
& +\left(-17+\frac{\sqrt{35}}{3}+\frac{4 \sqrt{42}}{13}+\frac{8 \sqrt{3}}{7}+\frac{8 \sqrt{10}}{13}+\frac{3 \sqrt{7}}{4}+\frac{24 \sqrt{8}}{17}\right) n \\
& +17-\frac{218 \sqrt{2}}{51}+\frac{\sqrt{15}}{4}-\frac{67 \sqrt{7}}{88}+\frac{\sqrt{35}}{3}+\frac{2 \sqrt{30}}{11}-\frac{14 \sqrt{42}}{13}-\frac{8 \sqrt{3}}{7}-\frac{20 \sqrt{10}}{13}+\frac{4 \sqrt{14}}{15}, \\
& E_{\mathrm{GA}_{5}}(G)=\log \left(\mathrm{GA}_{5}\right)-\frac{1}{\left(\mathrm{GA}_{5}\right)} \log \left[\left(\frac{2 \sqrt{2}}{3}\right)^{(2 \sqrt{2} / 3)}\right]-\frac{1}{\left(\mathrm{GA}_{5}\right)} \log \left\{\left(\frac{\sqrt{15}}{4}\right)^{(\sqrt{15} / 4)}-\frac{1}{\left(\mathrm{GA}_{5}\right)} \log [m+2 n+1]\right. \\
& -\frac{1}{\left(\mathrm{GA}_{5}\right)} \log \left[\left(\frac{4 \sqrt{7}}{11}\right)^{(4 \sqrt{7} / 11)}\right]-\frac{1}{\left(\mathrm{GA}_{5}\right)} \log \left[(2 n+2) \times\left(\frac{\sqrt{35}}{6}\right)^{(\sqrt{35} / 6)}\right]-\frac{1}{\left(\mathrm{GA}_{5}\right)} \log \left[\left(\frac{2 \sqrt{30}}{11}\right)^{(2 \sqrt{30} / 11)}\right] \\
& -\frac{1}{\left(\mathrm{GA}_{5}\right)} \log \left[(4 m+2 n-7) \times\left(\frac{2 \sqrt{42}}{13}\right)^{(2 \sqrt{42} / 13)}\right]-\frac{1}{\left(\mathrm{GA}_{5}\right)} \log \left[(2 n-2) \times\left(\frac{4 \sqrt{3}}{7}\right)^{(4 \sqrt{3} / 7)}\right] \\
& -\frac{1}{\left(\mathrm{GA}_{5}\right)} \log \left[(2 m+2 n-5) \times\left(\frac{4 \sqrt{10}}{13}\right)^{(4 \sqrt{10} / 13)}\right]-\frac{1}{\left(\mathrm{GA}_{5}\right)} \log \left[\left(\frac{4 \sqrt{14}}{15}\right)^{(4 \sqrt{14} / 15)}\right]-\frac{1}{\left(\mathrm{GA}_{5}\right)} \log [m+2 n-4] \\
& -\frac{1}{\left(\mathrm{GA}_{5}\right)} \log \left[(2 m+2 n-3) \times\left(\frac{3 \sqrt{7}}{8}\right)^{(3 \sqrt{7} / 8)}\right]-\frac{1}{\left(\mathrm{GA}_{5}\right)} \log \left[(2 m+4 n-7) \times\left(\frac{6 \sqrt{8}}{17}\right)^{(6 \sqrt{8} / 17)}\right] \\
& -\frac{1}{\left(\mathrm{GA}_{5}\right)} \log [(15 m n-14 m-21 n+20)] \text {. }
\end{aligned}
$$

\section{Crystallographic Structure of $\mathbf{S i}_{2} \mathbf{C}_{3}-I I[m, n]$}

The framework of $\mathrm{Si}_{2} \mathrm{C}_{3}-I I$ sheet contains only hexagonal rings. This framework is greater in energy than that of $\mathrm{Si}_{2} \mathrm{C}_{3}-I$ sheet. On the other hand, the composition of $\mathrm{Si}_{2} \mathrm{C}_{3}-I I$ is quite different from $\mathrm{Si}_{2} \mathrm{C}_{3}-I$ sheet as $\mathrm{Si}-\mathrm{Si}$ bonds can be viewed and the ratio of $\mathrm{Si}$ atoms making $\mathrm{Si}$ dimers is half. Also, $\mathrm{Si}_{2} \mathrm{C}_{3}-I I$ is more unstable than $\mathrm{Si}_{2} \mathrm{C}_{3}-I$. The anticipated minimal energy frameworks $\mathrm{Si}_{2} \mathrm{C}_{3}-I$ and $\mathrm{Si}_{2} \mathrm{C}_{3}-I I$ sheets have exceptional semiconducting axioms that could be employed for monoelectronic utilization. The mechanic strength of the silicon carbide sheets is also significant. It is widely known that graphene carries magnificent elastic properties having immense elastic constants. Former analyses have demonstrated that silicon carbide also holds well elastic axioms.

In Figure 3 [27, 28], $m$ denotes the total associated unit cells in each row while $n$ shows the total sum of associated rows with $m$ number of cells. Figure 4 illustrates the way in which cells are associated in a row and the way of association of rows with each other. Also, $\left|V\left(\mathrm{Si}_{2} \mathrm{C}_{3}-I I\right)\right|=10 \mathrm{mn}$ and $\left|E\left(\mathrm{Si}_{2} \mathrm{C}_{3}-I I\right)\right|=3(5 m n-n-m)$

3.1. Methodology of Silicon Carbide $\mathrm{Si}_{2} \mathrm{C}_{3}-I I[m, n]$ Formulas. For silicon carbide $\mathrm{Si}_{2} \mathrm{C}_{3}-I I[m, n]$, we join one unit cell with another unit cell and then continue this 


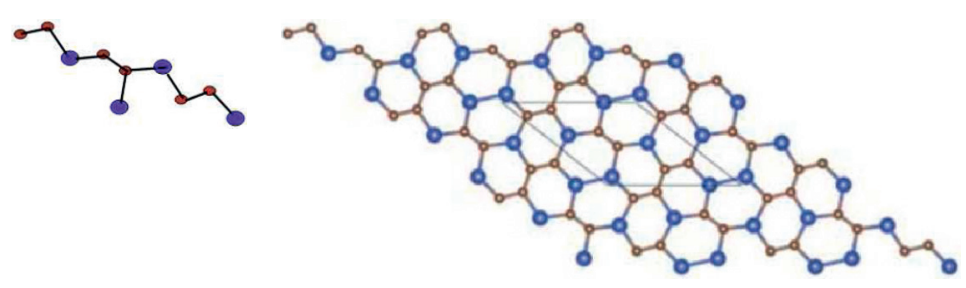

(a)

(b)

Figure 3: (a) Unit cell of $\mathrm{Si}_{2} \mathrm{C}_{3}-I I[m, n]$; (b) $\mathrm{Si}_{2} \mathrm{C}_{3}-I I[3,3]$.

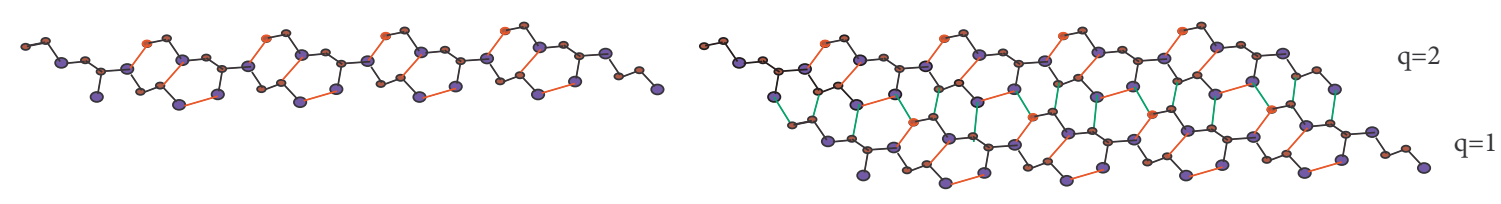

(a)

(b)

Figure 4: (a) $\mathrm{Si}_{2} \mathrm{C}_{3}-I I[5,1]$; (b) $\mathrm{Si}_{2} \mathrm{C}_{3}-I I[5,2]$.

TABLE 4: Vertex partition of $\mathrm{Si}_{2} \mathrm{C}_{3}-I I[m, n]$ established on degrees of each vertex.

\begin{tabular}{lcc}
\hline$\tau(m)$ & No. of repetitions & Kinds of vertices \\
\hline 1 & 3 & $M_{V_{1}}$ \\
2 & $6 m+6 n-6$ & $M_{V_{2}}$ \\
3 & $10 m n-6 m-6 n+3$ & $M_{V_{3}}$ \\
\hline
\end{tabular}

TABLE 5: Edge partition of $\mathrm{Si}_{2} \mathrm{C}_{3}-I I[m, n]$ established on degrees of end vertices.

\begin{tabular}{lcc}
\hline$(\tau(r), \tau(s))$ & No. of repetitions & Kinds of edges \\
\hline$(2,1)$ & 2 & $M_{E_{1}}$ \\
$(3,1)$ & 1 & $M_{E_{2}}$ \\
$(2,2)$ & $2 m+2 n$ & $M_{E_{3}}$ \\
$(3,2)$ & $8 m+8 n-14$ & $M_{E_{4}}$ \\
$(3,3)$ & $15 m n-13 m-13 n+11$ & $M_{E_{5}}$ \\
\hline
\end{tabular}

process horizontally till $n$ unit cells. Similar technique will be applied for vertical direction. As a result, we will obtain the $\mathrm{Si}_{2} \mathrm{C}_{3}-I I[m, n]$ sheet; see Figure 3 . Moreover, Tables 4 and 5 are used for computation of vertices and edges, respectively.

The edge partition of $\mathrm{Si}_{2} \mathrm{C}_{3}-I I[m, n]$ established on the addition of degree of terminal vertices of every edge is illustrated in Table 6.
TABle 6: Edge partition of $\mathrm{Si}_{2} \mathrm{C}_{3}-I I[m, n], m, n, s \geq 2$.

\begin{tabular}{lc}
\hline$\left(S_{s}, S_{t}\right)$ & Frequency \\
\hline$(3,2)$ & 2 \\
$(7,3)$ & 1 \\
$(4,3)$ & 2 \\
$(5,4)$ & 2 \\
$(5,5)$ & $-4+2(m+n)$ \\
$(7,5)$ & $2(m+n)$ \\
$(7,6)$ & $-2+2(m+n)$ \\
$(8,6)$ & $-6+2(m+n)$ \\
$(8,5)$ & $-6+(m+n)$ \\
$(8,7)$ & 2 \\
$(8,8)$ & $-8+2(m+n)$ \\
$(7,7)$ & 2 \\
$(9,7)$ & $-3+2(m+n)$ \\
$(9,8)$ & $-5+2(m+n)$ \\
$(9,9)$ & $23-19 n-19 m+15 m n$ \\
\hline
\end{tabular}

3.2. Computation of Entropies for Crystallographic Structure of $\mathrm{Si}_{2} \mathrm{C}_{3}-I I[m, n]$. This portion deals with the computation of topological indices and their corresponding graph entropies for the crystallographic structure of $\mathrm{Si}_{2} \mathrm{C}_{3}-I I[m, n]$.

3.2.1. The Randic Index and Randić Entropy for $\mathrm{Si}_{2} \mathrm{C}_{3}-I I[m, n]$. For $\alpha=1$, the Randić index and Randić entropy by Table 5 are

$$
\begin{aligned}
R_{1}\left(\mathrm{Si}_{2} \mathrm{C}_{3}-I I\right)= & 135 m n-61 m-61 n+22, \\
E_{R_{1}}\left(\mathrm{Si}_{2} \mathrm{C}_{3}-I I\right)= & \log [135 m n-61 m-61 n+22]-\frac{\log \left[(2) \times(2)^{(2)}\right]}{[135 m n-61 m-61 n+22]}-\frac{\log \left[(1) \times(3)^{(3)}\right]}{[135 m n-61 m-61 n+22]} \\
& -\frac{\log \left[(2 m+2 n) \times(4)^{(4)}\right]}{[135 m n-61 m-61 n+22]}-\frac{\log \left[(8 m+8 n-14) \times(6)^{(6)}\right]}{[135 m n-61 m-61 n+22]}-\frac{\log \left[(15 m n-13 m-13 n+11) \times(9)^{(9)}\right]}{[135 m n-61 m-61 n+22]} .
\end{aligned}
$$


For $\alpha=-1$, by using Table 5 , the Randić index and Randić entropy are $R_{-1}\left(\mathrm{Si}_{2} \mathrm{C}_{3}-I I\right)=(5 / 3) m n+(7 / 18) m+$ $(7 / 18) n+(2 / 9)$ and

$$
\begin{aligned}
E_{R_{-1}}\left(\mathrm{Si}_{2} \mathrm{C}_{3}-I I\right)= & \log \left(\frac{5}{3} m n+\frac{7}{18} m+\frac{7}{18} n+\frac{2}{9}\right)-\frac{\log \left[(2) \times(2)^{(-1 / 2)}\right]}{((5 / 3) m n+(7 / 18) m+(7 / 18) n+(2 / 9))} \\
& -\frac{\log \left[(1) \times(3)^{(-1 / 3)}\right]}{((5 / 3) m n+(7 / 18) m+(7 / 18) n+(2 / 9))}-\frac{\log \left[(2 m+2 n) \times(4)^{(-1 / 4)}\right]}{((5 / 3) m n+(7 / 18) m+(7 / 18) n+(2 / 9))} \\
& -\frac{\log \left[(8 m+8 n-14) \times(6)^{(-1 / 6)}\right]}{((5 / 3) m n+(7 / 18) m+(7 / 18) n+(2 / 9))}-\frac{\log \left[(15 m n-13 m-13 n+11) \times(9)^{(-1 / 9)}\right]}{((5 / 3) m n+(7 / 18) m+(7 / 18) n+(2 / 9))} .
\end{aligned}
$$

For $\alpha=1 / 2$, by using Table 2 , the Randić index and Randić entropy are $R_{1 / 2}\left(\mathrm{Si}_{2} \mathrm{C}_{3}-I\right)=45 m n+$ $(8 \sqrt{6}-35)(m+n)+2 \sqrt{2}+\sqrt{3}-14 \sqrt{6}+33$ and

$$
\begin{aligned}
E_{R_{1}}\left(\mathrm{Si}_{2} \mathrm{C}_{3}-I I\right)= & \log (45 m n+(8 \sqrt{6}-35)(m+n)+2 \sqrt{2}+\sqrt{3}-14 \sqrt{6}+33) \\
& -\frac{\log \left[(2) \times(2)^{(\sqrt{2} / 2)]}\right.}{(45 m n+(8 \sqrt{6}-35)(m+n)+2 \sqrt{2}+\sqrt{3}-14 \sqrt{6}+33)} \\
& -\frac{\log \left[(1) \times(3)^{(\sqrt{3} / 2)}\right]}{(45 m n+(8 \sqrt{6}-35)(m+n)+2 \sqrt{2}+\sqrt{3}-14 \sqrt{6}+33)} \\
& -\frac{\log \left[(2 m+2 n) \times(4)^{(\sqrt{4} / 2)}\right]}{(45 m n+(8 \sqrt{6}-35)(m+n)+2 \sqrt{2}+\sqrt{3}-14 \sqrt{6}+33)} \\
& -\frac{\log \left[(8 m+8 n-14) \times(6)^{(\sqrt{6} / 2)}\right]}{(45 m n+(8 \sqrt{6}-35)(m+n)+2 \sqrt{2}+\sqrt{3}-14 \sqrt{6}+33)} \\
& -\frac{\log [(15 m n-13 m-13 n+11) \times(9)(\sqrt{9} / 2)]}{(45 m n+(8 \sqrt{6}-35)(m+n)+2 \sqrt{2}+\sqrt{3}-14 \sqrt{6}+33)} .
\end{aligned}
$$

For $\alpha=-1 / 2$, by using Table 2 , the Randić index and Randić entropy are

$$
\begin{aligned}
R_{-1 / 2}\left(\mathrm{Si}_{2} \mathrm{C}_{3}-I I\right)= & 5 m n+((8 / \sqrt{6})-(10 / 3))(m+n)+\sqrt{2}+(1 / \sqrt{3})-(14 / \sqrt{6})+(11 / 3), \\
E_{R_{(-1 / 2)}}\left(\mathrm{Si}_{2} \mathrm{C}_{3}-I I\right)= & \log \left(5 m n+\left(\frac{8}{\sqrt{6}}-\frac{10}{3}\right)(m+n)+\sqrt{2}+\frac{1}{\sqrt{3}}-\frac{14}{\sqrt{6}}+\frac{11}{3}\right) \\
& -\frac{\log \left[(2) \times(2)^{(-1 / 2 \sqrt{2})}\right]}{(5 m n+((8 / \sqrt{6})-(10 / 3))(m+n)+\sqrt{2}+(1 / \sqrt{3})-(14 / \sqrt{6})+(11 / 3)}
\end{aligned}
$$




$$
\begin{gathered}
-\frac{\log \left[(1) \times(3)^{(-1 / 2 \sqrt{3})}\right]}{(5 m n+((8 / \sqrt{6})-(10 / 3))(m+n)+\sqrt{2}+(1 / \sqrt{3})-(14 / \sqrt{6})+(11 / 3)} \\
-\frac{\log \left[(2 m+2 n) \times(4)^{(-1 / 2 \sqrt{4})}\right]}{(5 m n+((8 / \sqrt{6})-(10 / 3))(m+n)+\sqrt{2}+(1 / \sqrt{3})-(14 / \sqrt{6})+(11 / 3)} \\
-\frac{\log \left[(8 m+8 n-14) \times(6)^{(-1 / 2 \sqrt{6})}\right]}{(5 m n+((8 / \sqrt{6})-(10 / 3))(m+n)+\sqrt{2}+(1 / \sqrt{3})-(14 / \sqrt{6})+(11 / 3)} \\
-\frac{\log \left[(15 m n-13 m-13 n+11) \times(9)^{(-1 / 2 \sqrt{9})}\right]}{(5 m n+((8 / \sqrt{6})-(10 / 3))(m+n)+\sqrt{2}+(1 / \sqrt{3})-(14 / \sqrt{6})+(11 / 3)} .
\end{gathered}
$$

3.2.2. The $A B C$ Index and $A B C$ Entropy of $S i_{2} C_{3}-I I[m, n] . \quad$ By using Table 5 , the $A B C$ index and $A B C$ entropy are

$$
\begin{aligned}
A B C\left(\mathrm{Si}_{2} \mathrm{C}_{3}-I I\right)= & 10 m n+((10 / \sqrt{2})-(26 / 3))(m+n)-(12 / \sqrt{2})+\sqrt{(2 / 3)}+(22 / 3), \\
E_{A B C}\left(\mathrm{Si}_{2} \mathrm{C}_{3}-I I\right)= & \log \left(A B C\left(\mathrm{Si}_{2} \mathrm{C}_{3}-I I\right)\right)-\frac{\log \left[(2) \times(1 / \sqrt{2})^{(1 / \sqrt{2})}\right]}{\left(A B C\left(\mathrm{Si}_{2} \mathrm{C}_{3}-I I\right)\right)}-\frac{\log \left[(15 m n-13 m-13 n+11) \times(2 / 3)^{(2 / 3)}\right]}{\left(A B C\left(\mathrm{Si}_{2} \mathrm{C}_{3}-I I\right)\right)} \\
& -\frac{\log \left[(2 m+2 n) \times(1 / \sqrt{2})^{(1 / \sqrt{2})}\right]}{\left(A B C\left(\mathrm{Si}_{2} \mathrm{C}_{3}-I I\right)\right)}-\frac{\log \left[(8 m+8 n-14) \times(1 / \sqrt{2})^{(1 / \sqrt{2})}\right]}{\left(A B C\left(\mathrm{Si}_{2} \mathrm{C}_{3}-I I\right)\right)}-\frac{\log \left[(1) \times(\sqrt{(2 / 3)})^{\sqrt{(2 / 3)}}\right]}{\left(A B C\left(\mathrm{Si}_{2} \mathrm{C}_{3}-I I\right)\right)} .
\end{aligned}
$$

3.2.3. The GA Index and GA Entropy of $\mathrm{Si}_{2} \mathrm{C}_{3}-I I[m, n] . \quad$ By using Table 5, the GA index and GA entropy are

$$
\begin{aligned}
\mathrm{GA}\left(\mathrm{Si}_{2} \mathrm{C}_{3}-I I\right)= & 15 m n+((16 \sqrt{6} / 5)-11)(m+n)+(4 \sqrt{2} / 3)+(\sqrt{3} / 2)-(28 \sqrt{6} / 5)+11 \\
E_{\mathrm{GA}}\left(\mathrm{Si}_{2} \mathrm{C}_{3}-I I\right)= & \log \left(\mathrm{GA}\left(\mathrm{Si}_{2} \mathrm{C}_{3}-I I\right)\right)-\frac{\log \left[(2) \times[2 \sqrt{2} / 3]^{[2 \sqrt{2} / 3]}\right]}{\left(\mathrm{GA}\left(\mathrm{Si}_{2} \mathrm{C}_{3}-I I\right)\right)}-\frac{\log \left[[\sqrt{3} / 2]^{[\sqrt{3} / 2]}\right]}{\left(\mathrm{GA}\left(\mathrm{Si}_{2} \mathrm{C}_{3}-I I\right)\right)}-\frac{\log [2 m+2 n]}{\left(\mathrm{GA}\left(\mathrm{Si}_{2} \mathrm{C}_{3}-I I\right)\right)} \\
& -\frac{\log [(15 m n-13 m-13 n+11)]}{\left(\mathrm{GA}\left(\mathrm{Si}_{2} \mathrm{C}_{3}-I I\right)\right)} .
\end{aligned}
$$

3.2.4. The First Zagreb Index and First Zagreb Entropy of $\mathrm{Si}_{2} \mathrm{C}_{3}-I I[m, n]$. By using Table 5, the first Zagreb index and entropy are $M_{1}\left(\mathrm{Si}_{2} \mathrm{C}_{3}-I I\right)=90 m n-30 m-30 n+6$ and

$$
\begin{aligned}
E_{M_{1}}\left(\mathrm{Si}_{2} \mathrm{C}_{3}-I I\right)= & \log (90 m n-30 m-30 n+6)-\frac{\log [54]}{(90 m n-30 m-30 n+6)}-\frac{\log [256]}{(90 m n-30 m-30 n+6)} \\
& -\frac{\log [256(2 m+2 n)]}{(90 m n-30 m-30 n+6)}-\frac{\log [3125(8 m+8 n-14)]}{(90 m n-30 m-30 n+6)}-\frac{\log [46656(15 m n-13 m-13 n+11)]}{(90 m n-30 m-30 n+6)} .
\end{aligned}
$$

3.2.5. The Second Zagreb Index and Second Zagreb Entropy of $\mathrm{Si}_{2} \mathrm{C}_{3}-I I[m, n]$. By using Table 3, the second Zagreb index and second Zagreb entropy are $M_{2}\left(\mathrm{Si}_{2} \mathrm{C}_{3}-I I\right)=135 m n-$ $61 m-61 n+22$ and 


$$
\begin{aligned}
E_{M_{2}}\left(\mathrm{Si}_{2} \mathrm{C}_{3}-I I\right)= & \log (135 m n-61 m-61 n+22)-\frac{\log [8]}{(135 m n-61 m-61 n+22)}-\frac{\log [27]}{(135 m n-61 m-61 n+22)} \\
& -\frac{\log \left[(2 m+2 n) \times(4)^{(4)}\right]}{(135 m n-61 m-61 n+22)}-\frac{\log \left[(8 m+8 n-14) \times(5)^{(5)}\right]}{(135 m n-61 m-61 n+22)}-\frac{\log \left[(15 m n-13 m-13 n+11) \times(9)^{(9)}\right]}{(135 m n-61 m-61 n+22)}
\end{aligned}
$$

3.2.6. The Hyper-Zagreb Index and Hyper-Zagreb Entropy of $\mathrm{Si}_{2} \mathrm{C}_{3}-I I[m, n]$. By using Table 5, the hyper-Zagreb index and hyper-Zagreb entropy are

$$
\begin{aligned}
\operatorname{HM}\left(\mathrm{Si}_{2} \mathrm{C}_{3}-I I\right)= & 540 m n-236(m+n)+71 \\
E_{\mathrm{HM}}\left(\mathrm{Si}_{2} \mathrm{C}_{3}-I I\right)= & \log (540 m n-236(m+n)+71)-\frac{\log \left[(2) \times(9)^{(9)}\right]}{(540 m n-236(m+n)+71)}-\frac{\log \left[(m+8 n-14) \times(25){ }^{(25)}\right]}{(540 m n-236(m+n)+71)} \\
& -\frac{\log \left[(2 m+2 n) \times 16^{16}\right]}{(540 m n-236(m+n)+71)}-\frac{\log \left[16^{16}\right]}{(540 m n-236(m+n)+71)}-\frac{\log \left[36^{36}(15 m n-13 m-13 n+11)\right]}{(540 m n-236(m+n)+71)} .
\end{aligned}
$$

3.2.7. The Forgotten Index and Forgotten Entropy for $\mathrm{Si}_{2} \mathrm{C}_{3}-I I[m, n]$. By using Table 5 , the forgotten index and forgotten entropy are

$$
\begin{aligned}
F\left(\mathrm{Si}_{2} \mathrm{C}_{3}-I I\right)= & 270 m n-114(m+n)+36, \\
E_{F}\left(\mathrm{Si}_{2} \mathrm{C}_{3}-I I\right)= & \log (270 m n-114(m+n)+36)-\frac{\log \left[(2) \times(5)^{(5)}\right]}{(270 m n-114(m+n)+36)} \\
& -\frac{\log \left[10^{10}\right]}{(270 m n-114(m+n)+36)}-\frac{\log \left[(2 m+2 n) \times 8^{8}\right]}{(270 m n-114(m+n)+36)} \\
& -\frac{\log \left[(8 m+8 n-14) \times 13^{13}\right]}{(270 m n-114(m+n)+36)}-\frac{\log \left[(15 m n-13 m-13 n+11) \times 18^{18}\right]}{(270 m n-114(m+n)+36)} .
\end{aligned}
$$

3.2.8. The Augmented Zagreb Index and Augmented Zagreb Entropy of $\mathrm{Si}_{2} \mathrm{C}_{3}-I I[m, n]$. By using Table 5, it is easy to see that the augmented Zagreb index and augmented Zagreb entropy are

$$
\begin{aligned}
\mathrm{AZI}\left(\mathrm{Si}_{2} \mathrm{C}_{3}-I I\right)= & (10935 / 64) m n-(4357 / 64) m-(4357 / 64) n+(2091 / 64) \\
E_{\mathrm{AZI}}\left(\mathrm{Si}_{2} \mathrm{C}_{3}-I I\right)= & \log \left(\frac{10935}{64} m n-\frac{4357}{64} m-\frac{4357}{64} n+\frac{2091}{64}\right)-\frac{\log \left[(2) \times(8)^{(8)}\right]}{((10935 / 64) m n-(4357 / 64) m-(4357 / 64) n+(2091 / 64))} \\
& -\frac{\log \left[(1) \times[27 / 8]^{[27 / 8]}\right]}{((10935 / 64) m n-(4357 / 64) m-(4357 / 64) n+(2091 / 64))} \\
& -\frac{\log \left[(2 m+2 n) \times(8)^{(8)}\right]}{((10935 / 64) m n-(4357 / 64) m-(4357 / 64) n+(2091 / 64))}
\end{aligned}
$$




$$
-\frac{\log \left[(8 m+8 n-14) \times(8)^{(8)}\right]}{((10935 / 64) m n-(4357 / 64) m-(4357 / 64) n+(2091 / 64))}-\frac{\log \left[(15 m n-13 m-13 n+11) \times\left(9^{3} / 4^{3}\right)^{\left(9^{3} / 4^{3}\right)}\right]}{((10935 / 64) m n-(4357 / 64) m-(4357 / 64) n+(2091 / 64))} .
$$

3.2.9. The Balaban Index and Balaban Entropy for $\mathrm{Si}_{2} \mathrm{C}_{3}-I I[m, n]$. By using Table 2 , it is easy to see that the Balaban index and Balaban entropy are

$$
\begin{aligned}
J\left(\mathrm{Si}_{2} \mathrm{C}_{3}-I I\right)= & \frac{15 m n-3(m+n)}{5 m n-3(m+n)+2}\left[\sqrt{2}+\frac{1}{\sqrt{3}}+(m+n)+\frac{1}{\sqrt{6}}(8 m+8 n-14)+\frac{1}{3}(15 m n-13 m-13 n+11)\right] \\
E_{J}\left(\mathrm{Si}_{2} \mathrm{C}_{3}-I I\right)= & \log \left(J\left(\mathrm{Si}_{2} \mathrm{C}_{3}-I I\right)\right)-\frac{\log \left[(2) \times[q / \sqrt{2}(q-p+2)]^{[q / \sqrt{2}(q-p+2)]}\right]}{\left(J\left(\mathrm{Si}_{2} \mathrm{C}_{3}-I I\right)\right)}-\frac{\log [(1) \times[q / \sqrt{3}(q-p+2)][q / \sqrt{3}(q-p+2)]]}{\left(J\left(\mathrm{Si}_{2} \mathrm{C}_{3}-I I\right)\right)} \\
& -\frac{\log \left[(2 m+2 n) \times[q / 2(q-p+2)]^{[q / 2(q-p+2)]}\right]}{\left(J\left(\mathrm{Si}_{2} \mathrm{C}_{3}-I I\right)\right)}-\frac{\log [[(8 m+8 n-14) \times[q / \sqrt{6}(q-p+2)][q / \sqrt{6}(q-p+2)]}{(q)]} \\
& \left.\left.-\frac{\log \left[(15 m n-13 m-13 n+11) \times[q / 3(q-p+2)]^{[q / 3(q-p+2)]}\right]}{\left(J\left(\mathrm{Si}_{2} \mathrm{C}_{3}-I I\right)\right)} . I I\right)\right)
\end{aligned}
$$

3.2.10. The Redefined First Zagreb Index and Redefined First Zagreb Entropy for $\mathrm{Si}_{2} \mathrm{C}_{3}-I I[m, n]$. By using Table 5, the redefined first Zagreb index and redefined first Zagreb entropy are $\operatorname{ReZG}_{1}\left(\mathrm{Si}_{2} \mathrm{C}_{3}-I I\right)=10 m n$ and

$$
\begin{aligned}
\mathrm{ENT}_{\mathrm{ReZG}_{1}}\left(\mathrm{Si}_{2} \mathrm{C}_{3}-I I\right)= & \log (10 m n)-\frac{\log \left[(2) \times(3 / 2)^{(3 / 2)}\right]}{(10 m n)}-\frac{\log \left[(4 / 3)^{(4 / 3)}\right]}{(10 m n)}-\frac{\log [2 m+2 n]}{(10 m n)} \\
& -\frac{\log \left[(8 m+8 n-14) \times(5 / 6)^{(5 / 6)}\right]}{(10 m n)}-\frac{\log \left[(15 m n-13 m-13 n+11) \times(2 / 3)^{(2 / 3)}\right]}{(10 m n)} .
\end{aligned}
$$

3.2.11. The Redefined Second Zagreb Index and Redefined Second Zagreb Entropy for $\mathrm{Si}_{2} \mathrm{C}_{3}-I I[m, n]$. By using Table 5, the redefined second Zagreb index and redefined second Zagreb entropy are $\operatorname{ReZG}_{2}\left(\mathrm{Si}_{2} \mathrm{C}_{3}-I I\right)=(45$ $m n / 3)-(79(m+n) / 10)+(107 / 60)$ and

$$
\begin{aligned}
E_{\mathrm{ReZG}_{2}}\left(\mathrm{Si}_{2} \mathrm{C}_{3}-I I\right)= & \log \left(\frac{45}{3} m n-\frac{79}{10} m-\frac{79}{10} n+\frac{107}{60}\right)-\frac{\log \left[(2) \times(2 / 3)^{(2 / 3)}\right]}{((45 / 3) m n-(79 / 10) m-(79 / 10) n+(107 / 60))} \\
& -\frac{\log \left[(1) \times(3 / 4)^{(3 / 4)}\right]}{((45 / 3) m n-(79 / 10) m-(79 / 10) n+(107 / 60))}-\frac{\log [(2 m+2 n) \times(1)]}{((45 / 3) m n-(79 / 10) m-(79 / 10) n+(107 / 60))} \\
& -\frac{\log \left[(8 m+8 n-14) \times(6 / 5)^{(6 / 5)}\right]}{((45 / 3) m n-(79 / 10) m-(79 / 10) n+(107 / 60))}-\frac{\log \left[(15 m n-13 m-13 n+11) \times(3 / 2)^{(3 / 2)}\right]}{((45 / 3) m n-(79 / 10) m-(79 / 10) n+(107 / 60))}
\end{aligned}
$$


3.2.12. The Redefined Third Zagreb Index and Redefined Third Zagreb Entropy for $\mathrm{Si}_{2} \mathrm{C}_{3}-I I[m, n]$. By using Table 5, the redefined third Zagreb index and redefined third Zagreb entropy are

$$
\begin{aligned}
\operatorname{ReZG}_{3}\left(\mathrm{Si}_{2} \mathrm{C}_{3}-I I\right)= & 810 m n-430(m+n)+198 \\
E_{\mathrm{ReZG}_{3}}\left(\mathrm{Si}_{2} \mathrm{C}_{3}-I I\right)= & \log (810 m n-430 m-430 n+198)-\frac{\log \left[(2) \times(6)^{(6)}\right]}{(810 m n-430 m-430 n+198)} \\
& -\frac{\log \left[(1) \times(12)^{(12)}\right]}{(810 m n-430 m-430 n+198)}-\frac{\log \left[(2 m+2 n) \times(16)^{(16)}\right]}{(810 m n-430 m-430 n+198)} \\
& -\frac{\log \left[(8 m+8 n-14) \times(30)^{(30)}\right]}{(810 m n-430 m-430 n+198)}-\frac{\log \left[(15 m n-13 m-13 n+11) \times(54)^{(54)}\right]}{(810 m n-430 m-430 n+198)} .
\end{aligned}
$$

3.2.13. The Fourth $A B C$ Index and Fourth $A B C$ Entropy of $\mathrm{Si}_{2} \mathrm{C}_{3}-I I[m, n]$. By using Table 6, the fourth $A B C$ index and fourth $A B C$ entropy are

$$
\begin{aligned}
& A B C_{4}\left(\mathrm{Si}_{2} \mathrm{C}_{3}-I I\right)=\frac{23 m n}{3}+(m+n)\left[\frac{22 \sqrt{2}}{15}+2\left(\sqrt{\frac{2}{7}}\right)+2\left(\sqrt{\frac{11}{42}}\right)+\left(\sqrt{\frac{11}{10}}\right)+\frac{\sqrt{14}}{4}+\frac{2 \sqrt{2}}{3}+\left(\sqrt{\frac{5}{6}}-\frac{67}{9}\right)\right] \\
& +2\left(\sqrt{\frac{2}{21}}\right)+\left(\sqrt{\frac{5}{3}}\right)+2\left(\sqrt{\frac{7}{5}}\right)-\frac{8 \sqrt{2}}{5}-2\left(\sqrt{\frac{11}{42}}\right)-3\left(\sqrt{\frac{11}{10}}\right)+2\left(\sqrt{\frac{13}{56}}\right)-\sqrt{14}+\frac{4 \sqrt{3}}{7} \\
& -\frac{5}{2}\left(\sqrt{\frac{5}{6}}\right)+\frac{65}{9} \\
& \mathrm{ENT}_{A B C_{4}}(G)=\log \left(A B C_{4}\right)-\frac{1}{\left(A B C_{4}\right)} \log \left[(2) \times\left(\frac{1}{\sqrt{2}}\right)^{(1 / \sqrt{2})}\right]-\frac{1}{\left(A B C_{4}\right)} \log \left[(2 m+2 n-4) \times\left(\frac{2 \sqrt{2}}{5}\right)^{(2 \sqrt{2} / 5)}\right] \\
& -\frac{1}{\left(A B C_{4}\right)} \log \left[(2) \times\left(\frac{\sqrt{5}}{2 \sqrt{3}}\right)^{(\sqrt{5} / 2 \sqrt{3})}\right]-\frac{1}{\left(A B C_{4}\right)} \log \left[(2) \times\left(\frac{\sqrt{7}}{2 \sqrt{5}}\right)^{(\sqrt{7} / 2 \sqrt{5})}\right]-\frac{1}{\left(A B C_{4}\right)} \log \left[\left(\frac{2 \sqrt{2}}{\sqrt{21}}\right)^{(2 \sqrt{2} / \sqrt{21})}\right] \\
& -\frac{1}{\left(A B C_{4}\right)} \log \left[(2 m+2 n) \times\left(\frac{\sqrt{2}}{\sqrt{7}}\right)^{(\sqrt{2} / \sqrt{7})}\right]-\frac{1}{\left(A B C_{4}\right)} \log \left[(2 m+2 n-2) \times\left(\frac{\sqrt{11}}{\sqrt{42}}\right)^{(\sqrt{11} / \sqrt{42})}\right] \\
& -\frac{1}{\left(A B C_{4}\right)} \log \left[(2 m+2 n-6) \times\left(\frac{1}{2}\right)^{(1 / 2)}\right]-\frac{1}{\left(A B C_{4}\right)} \log \left[(2 m+2 n-6) \times\left(\frac{\sqrt{11}}{\sqrt{40}}\right)^{(\sqrt{11} / \sqrt{40})}\right] \\
& -\frac{1}{\left(A B C_{4}\right)} \log \left[(2) \times\left(\frac{\sqrt{13}}{\sqrt{56}}\right)^{(\sqrt{13} / \sqrt{56})}\right]-\frac{1}{\left(A B C_{4}\right)} \log \left[(2 m+2 n-8) \times\left(\frac{\sqrt{14}}{8}\right)^{(\sqrt{14} / 8)}\right] \\
& -\frac{1}{\left(A B C_{4}\right)} \log \left[(2) \times\left(\frac{2 \sqrt{3}}{7}\right)^{(2 \sqrt{3} / 7)}\right]-\frac{1}{\left(A B C_{4}\right)} \log \left[(2 m+2 n-3) \times\left(\frac{\sqrt{2}}{3}\right)^{(\sqrt{2} / 3)}\right] \\
& -\frac{1}{\left(A B C_{4}\right)} \log \left[\left[(2 m+2 n-5) \times\left(\frac{\sqrt{5}}{2 \sqrt{6}}\right)^{(\sqrt{5} / 2 \sqrt{6})}\right]-\frac{1}{\left(A B C_{4}\right)} \log \left[(15 m n-19 m-19 n+23) \times\left(\frac{4}{9}\right)^{(4 / 9)}\right]\right. \text {. }
\end{aligned}
$$


3.2.14. The Fifth GA Index and Fifth GA Entropy of $\mathrm{Si}_{2} \mathrm{C}_{3}-I I[m, n]$. By using Table 6 , the fifth GA index and fifth GA entropy are

$$
\begin{aligned}
& \mathrm{GA}_{5}(G)=15 m n+(m+n)\left[-15+\frac{\sqrt{35}}{3}+\frac{4 \sqrt{42}}{13}+\frac{8 \sqrt{3}}{7}+\frac{8 \sqrt{10}}{13}+\frac{3 \sqrt{7}}{4}+\frac{24 \sqrt{2}}{17}\right]+\frac{4 \sqrt{6}}{5} \\
& +\frac{\sqrt{21}}{5}+\frac{8 \sqrt{3}}{7}+\frac{8 \sqrt{5}}{9}-\frac{4 \sqrt{42}}{13}-\frac{24 \sqrt{3}}{7}-\frac{24 \sqrt{10}}{13}+\frac{8 \sqrt{14}}{15}-\frac{9 \sqrt{7}}{8}-\frac{60 \sqrt{2}}{17}+13 \text {, } \\
& E_{\mathrm{GA}_{5}}(G)=\log \left(\mathrm{GA}_{5}\right)-\frac{1}{\left(\mathrm{GA}_{5}\right)} \log \left[(2) \times\left(\frac{2 \sqrt{6}}{5}\right)^{(2 \sqrt{6} / 5)}\right]-\frac{1}{\left(\mathrm{GA}_{5}\right)} \log \left[\left(\frac{\sqrt{21}}{5}\right)^{(\sqrt{21} / 5)}\right]-\frac{1}{\left(\mathrm{GA}_{5}\right)} \log \left[(2) \times\left(\frac{4 \sqrt{3}}{7}\right)^{(4 \sqrt{3} / 7)}\right] \\
& -\frac{1}{\left(\mathrm{GA}_{5}\right)} \log \left[(2) \times\left(\frac{4 \sqrt{5}}{9}\right)^{(4 \sqrt{5} / 9)}\right]-\frac{1}{\left(\mathrm{GA}_{5}\right)} \log [2 m+2 n-4]-\frac{1}{\left(\mathrm{GA}_{5}\right)} \log \left[(2 m+2 n) \times\left(\frac{\sqrt{35}}{6}\right)^{(\sqrt{35} / 6)}\right] \\
& -\frac{1}{\left(\mathrm{GA}_{5}\right)} \log \left[(2 m+2 n-2) \times\left(\frac{2 \sqrt{42}}{13}\right)^{(2 \sqrt{42} / 13)}\right]-\frac{1}{\left(\mathrm{GA}_{5}\right)} \log \left[(2 m+2 n-6) \times\left(\frac{4 \sqrt{3}}{7}\right)^{(4 \sqrt{3} / 7)}\right] \\
& -\frac{1}{\left(\mathrm{GA}_{5}\right)} \log \left[(2 m+2 n-6) \times\left(\frac{4 \sqrt{10}}{13}\right)^{(4 \sqrt{10} / 13)}\right]-\frac{1}{\left(\mathrm{GA}_{5}\right)} \log \left[(2) \times\left(\frac{4 \sqrt{14}}{15}\right)^{(4 \sqrt{14} / 15)}\right] \\
& -\frac{1}{\left(\mathrm{GA}_{5}\right)} \log [(2 m+2 n-8)]-\frac{1}{\left(\mathrm{GA}_{5}\right)} \log [2]-\frac{1}{\left(\mathrm{GA}_{5}\right)} \log \left[(2 m+2 n-3) \times\left(\frac{3 \sqrt{7}}{8}\right)^{(3 \sqrt{7} / 8)}\right] \\
& -\frac{1}{\left(\mathrm{GA}_{5}\right)} \log \left[(2 m+2 n-5) \times\left(\frac{12 \sqrt{2}}{7}\right)^{(12 \sqrt{2} / 7)}\right]-\frac{1}{\left(\mathrm{GA}_{5}\right)} \log [15 m n-19 m-19 n+23] \text {. }
\end{aligned}
$$

\section{Comparisons and Discussion for $\mathbf{S i}_{2} \mathbf{C}_{3}-I[m, n]$}

In this segment, we discussed many topological indices with their respective entropies. The quantitative and pictorial comparisons are illustrated in Tables 7-11 and Figures 5-12, respectively. Due to the complexity of molecular structures, different molecular descriptors in association with their entropy measures are used to anticipate the maximum structural information.

These descriptors possess a lot of chemical properties like Randić index which has good correlation between various physicochemical characteristics of alkanes such as retention times of chromatographic parameters in the Antoine equation for surface areas, vapor pressure, and boiling points, etc. Recently, the utilization of this index is related with medicine and pharmacological matters. Also $A B C$ index can be a useful prognostic tool in the investigation of the heat formation in alkanes. It is also associated with stability of linear and branched alkanes both quantitatively and qualitatively. Furthermore, the geometric arithmetic index provides better correlation coefficients as compared to other descriptors.

The Zagreb type indices are used to measure the total $\pi$-electron energy of the molecules [2]. In recent times, Zagreb indices along with their invariant have been employed to examine complexity and heterosystems [29]. These indices are also used for acquiring multilinear regression models as well as in QSPR and QSAR studies [30]. The forgotten index is useful in the analysis of pharmacological and chemical properties of medication atomic structures. The AZI index determines an excellent correlation of branched alkanes as well as linear alkanes. The strain energy of cycloalkanes is also estimated by this index. Furthermore, Balaban index is correlated with acute toxicity of mediums and variety of physicochemical properties. It is also helpful for transformation of heteroatoms. These indices are also used in designing the fractional bounds. Entropy function is monotonic as, in all cases, an increase of entropy measure is observed with the increase in the size of the molecular structure. 
TABLE 7: Comparison of Randić entropies for $\mathrm{Si}_{2} \mathrm{C}_{3}-I[m, n]$.

\begin{tabular}{llllr}
\hline$[m, n]$ & $E_{R_{1}}$ & $E_{R_{-1}}$ & $E_{R_{1 / 2}}$ & $E_{R_{-1 / 2}}$ \\
\hline$[2,2]$ & 2.48 & 0.46 & 2.20 & 1.16 \\
{$[3,3]$} & 2.94 & 1.03 & 2.49 & 1.57 \\
{$[4,4]$} & 3.23 & 1.33 & 2.77 & 1.84 \\
{$[5,5]$} & 3.45 & 1.55 & 3.99 & 2.06 \\
{$[6,6]$} & 3.62 & 1.72 & 3.30 & 2.23 \\
{$[7,7]$} & 3.76 & 1.87 & 3.45 & 2.37 \\
{$[8,8]$} & 3.89 & 1.99 & 3.53 & 2.49 \\
{$[9,9]$} & 3.99 & 2.11 & 3.62 & 2.59 \\
{$[10,10]$} & 4.09 & 2.20 & & 2.69 \\
\hline
\end{tabular}

TABLE 8: Comparison of $E_{A B C}, E_{\mathrm{GA}}, E_{M_{1}}$, and $E_{M_{2}}$ entropies for $\mathrm{Si}_{2} \mathrm{C}_{3}-I[m, n]$.

\begin{tabular}{lclll}
\hline$[m, n]$ & $E_{A B C}$ & $E_{\mathrm{GA}}$ & $E_{M_{1}}$ & 2.35 \\
\hline$[2,2]$ & 1.46 & 1.80 & 2.79 & \\
{$[3,3]$} & 1.86 & 2.17 & 3.08 & \\
{$[4,4]$} & 2.14 & 2.42 & 3.30 & 2.94 \\
{$[5,5]$} & 2.35 & 2.61 & 3.53 & 3.23 \\
{$[6,6]$} & 2.52 & 2.77 & 3.68 & 3.45 \\
{$[7,7]$} & 2.66 & 2.90 & 3.73 & 3.62 \\
{$[8,8]$} & 2.78 & 3.01 & 3.83 & 3.89 \\
{$[9,9]$} & 2.89 & 3.11 & 3.93 & 3.99 \\
{$[10,10]$} & 2.98 & 3.20 & & 4.11 \\
\hline
\end{tabular}

TABle 9: Comparison of $E_{\mathrm{HM}}, E_{F}, E_{\mathrm{AZI}}$, and $E_{J}$ entropies for $\mathrm{Si}_{2} \mathrm{C}_{3}-I[m, n]$.

\begin{tabular}{lllll}
\hline$[m, n]$ & $E_{\mathrm{HM}}$ & $E_{F}$ & $E_{\text {AZI }}$ & \\
\hline$[2,2]$ & 3.17 & 2.78 & 2.61 & \\
{$[3,3]$} & 3.58 & 3.24 & 3.06 & \\
{$[4,4]$} & 3.85 & 3.54 & 3.34 & 2.06 \\
{$[5,5]$} & 4.07 & 3.75 & 3.56 & 2.33 \\
{$[6,6]$} & 4.23 & 3.93 & 3.73 & 2.53 \\
{$[7,7]$} & 4.37 & 4.07 & 3.87 & 2.69 \\
{$[8,8]$} & 4.50 & 4.19 & 3.99 & 2.83 \\
{$[9,9]$} & 4.61 & 4.30 & 4.10 & 3.06 \\
{$[10,10]$} & 4.70 & 4.40 & 4.20 & 3.15 \\
\hline
\end{tabular}

TABLE 10: Comparison of the redefined Zagreb entropies for $\mathrm{Si}_{2} \mathrm{C}_{3}-I[m, n]$.

\begin{tabular}{lccc}
\hline$[m, n]$ & $E_{\mathrm{ReZG}_{1}}$ & $E_{\mathrm{ReZG}_{2}}$ & $E_{\mathrm{ReZG}_{3}}$ \\
\hline$[2,2]$ & 1.51 & 1.761 & 3.19 \\
{$[3,3]$} & 1.90 & 2.19 & 3.69 \\
{$[4,4]$} & 2.17 & 2.47 & 3.99 \\
{$[5,5]$} & 2.38 & 2.69 & 4.21 \\
{$[6,6]$} & 2.54 & 2.86 & 4.39 \\
{$[7,7]$} & 2.68 & 2.99 & 4.54 \\
{$[8,8]$} & 2.80 & 3.12 & 4.66 \\
{$[9,9]$} & 2.90 & 3.23 & 4.77 \\
{$[10,10]$} & 2.99 & 3.32 & 4.87 \\
\hline
\end{tabular}

\section{Comparisons and} Discussion for $\mathbf{S i}_{2} \mathbf{C}_{3}-I I[m, n]$

Physicochemical properties depend upon their molecular structures. Thus modeling and prediction of these physicochemical properties and biological activities is an important field of study. Physical organic chemistry will be intended for clarifying thoroughly how these properties are established by the structure. So in this consideration, one of the essential points is the choice of suitable topological descriptors embracing the information stored in the molecular structure. It is to be taken into consideration that due 
TABLE 11: Comparison of the $A B C_{4}$ and $\mathrm{GA}_{5}$ entropies for $\mathrm{Si}_{2} \mathrm{C}_{3}-I[m, n]$.

\begin{tabular}{lcr}
\hline$[m, n]$ & $E_{A B C_{4}}$ & $E_{G A_{5}}$ \\
\hline$[2,2]$ & 1.34 & 1.69 \\
{$[3,3]$} & 1.73 & 2.08 \\
{$[4,4]$} & 1.99 & 2.35 \\
{$[5,5]$} & 2.20 & 2.55 \\
{$[6,6]$} & 2.36 & 2.71 \\
{$[7,7]$} & 2.50 & 2.85 \\
{$[8,8]$} & 2.62 & 2.97 \\
{$[9,9]$} & 2.72 & 3.07 \\
{$[10,10]$} & 2.82 & 3.17 \\
\hline
\end{tabular}

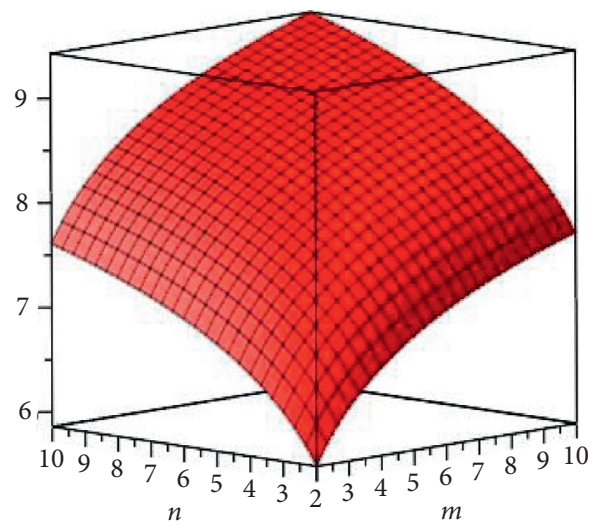

(a)

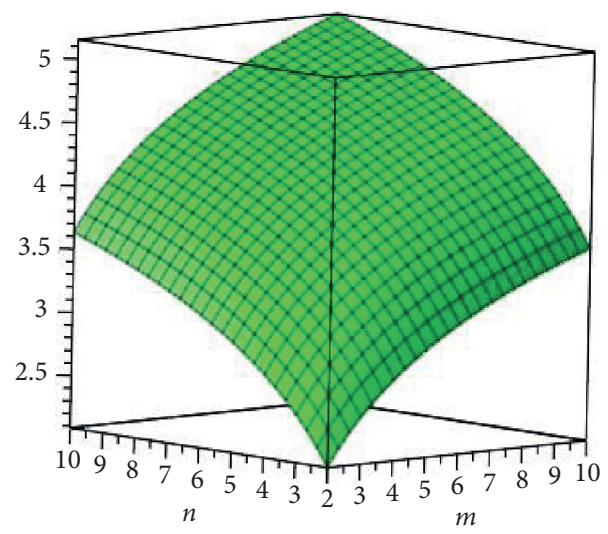

(b)

Figure 5: (a) Randić entropy for $\alpha=1$; (b) Randić entropy for $\alpha=-1$.

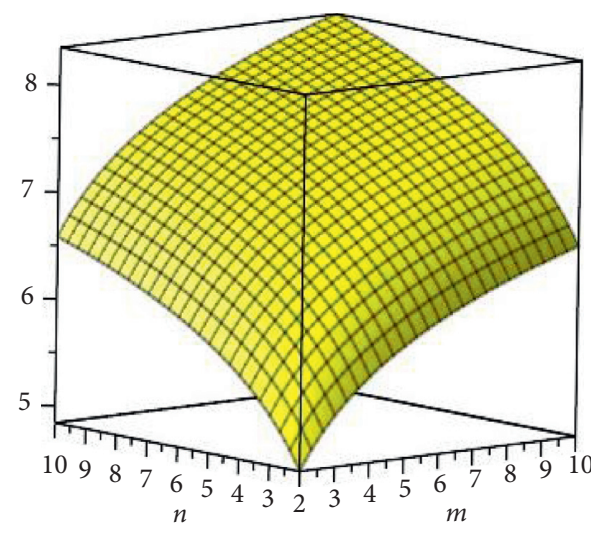

(a)

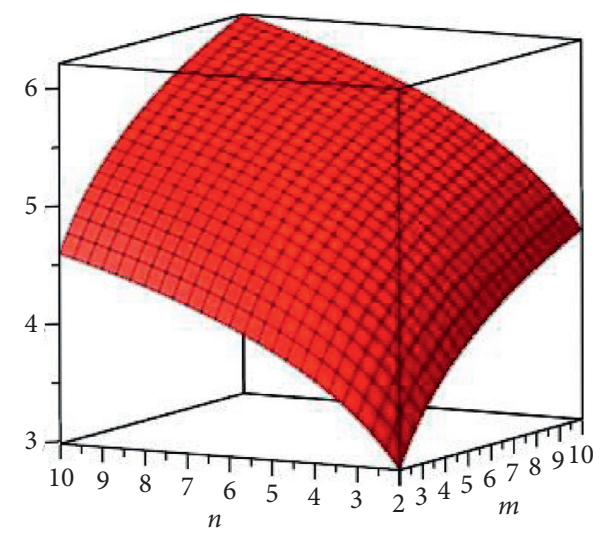

(b)

Figure 6: (a) Randić entropy for $\alpha=(1 / 2)$; (b) Randić entropy for $\alpha=(-1 / 2)$.

to the complexity of these molecular structures, it is no possible to anticipate that a single descriptor would be able to contain all the structural information. This is the foremost purpose why the study for molecular structure descriptors remains through a usual procedure grounded on the required characteristics that a molecular structure descriptor requires to possess.
In this section, we estimated degree-based entropy measures. In [31, 32], many algorithms were recommended to examine the structural complexity. But the entropy approach is reviewed to be the most substantial approach to distinguish the structural information of the complex networks. Therefore, we have listed mathematically some degree-based entropies for small considerations of parameters 


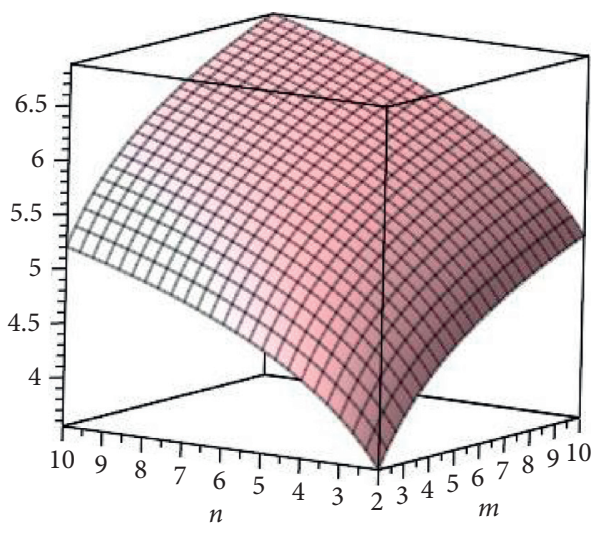

(a)

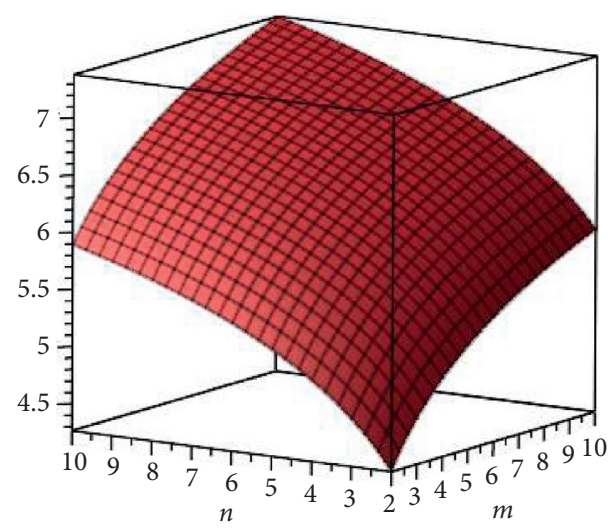

(b)

FIgURE 7: (a) The atom bond connectivity entropy; (b) the geometric arithmetic entropy.

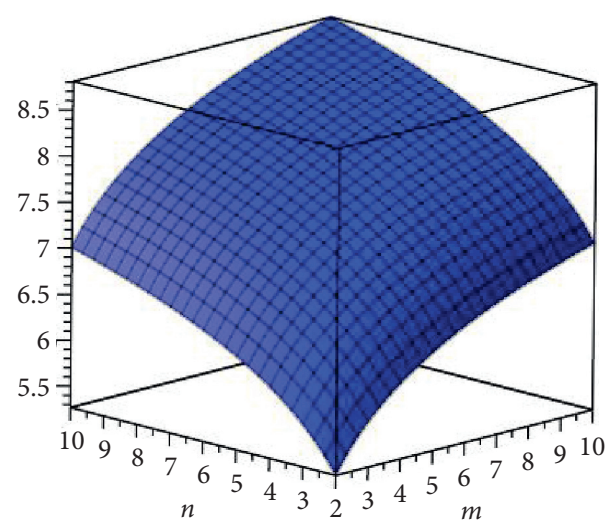

(a)

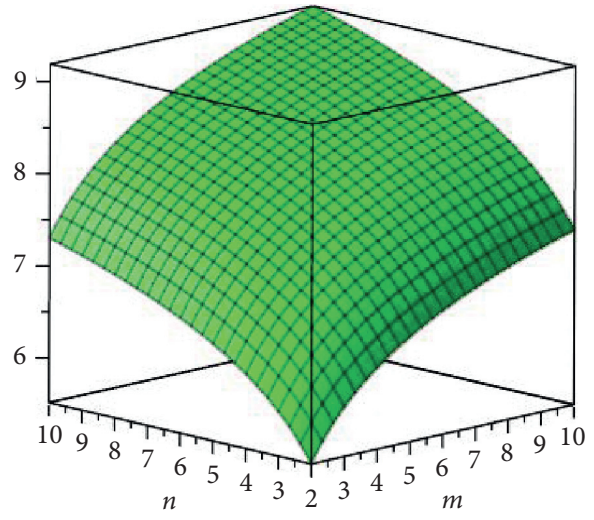

(b)

Figure 8: (a) The first Zagreb entropy; (b) the second Zagreb entropy.

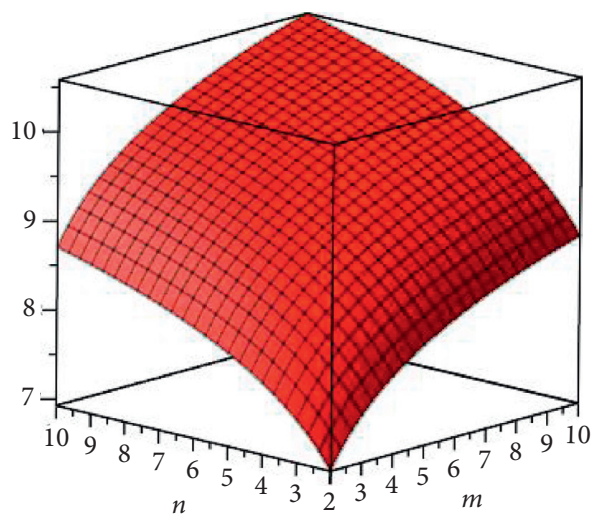

(a)

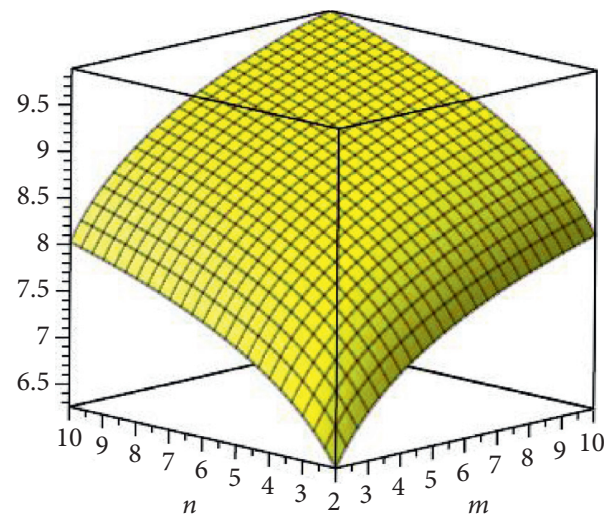

(b)

Figure 9: (a) The hyper-Zagreb entropy; (b) the forgotten entropy. 


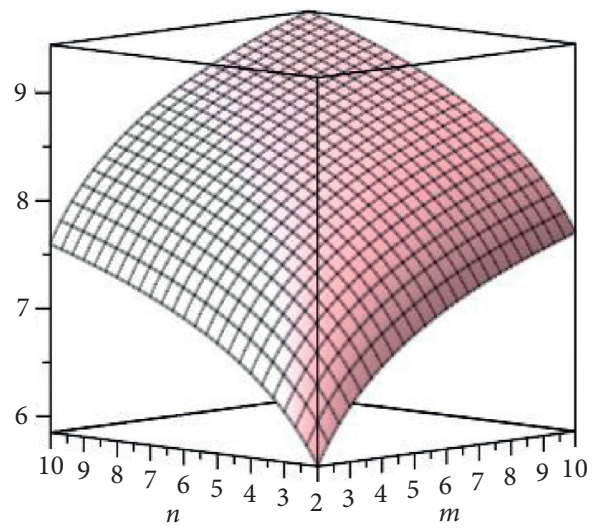

(a)

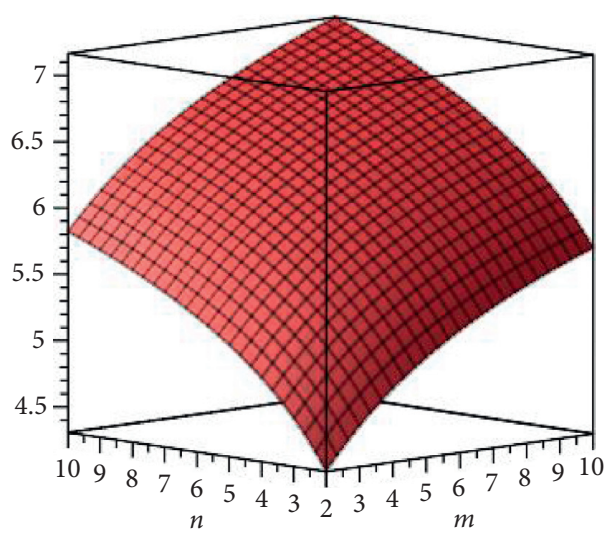

(b)

Figure 10: (a) The augmented Zagreb entropy; (b) the Balaban entropy.

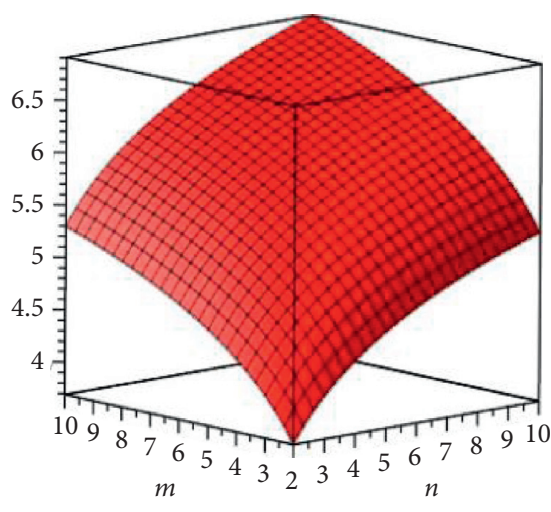

(a)

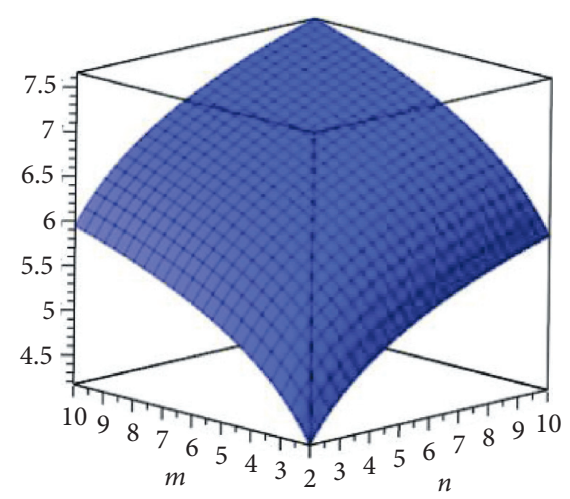

(b)

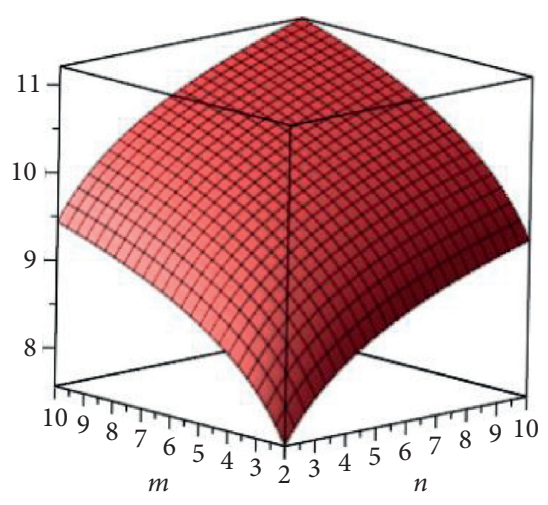

(c)

Figure 11: (a) The redefined first Zagreb entropy; (b) the redefined second Zagreb entropy; (c) the redefined third Zagreb entropy.

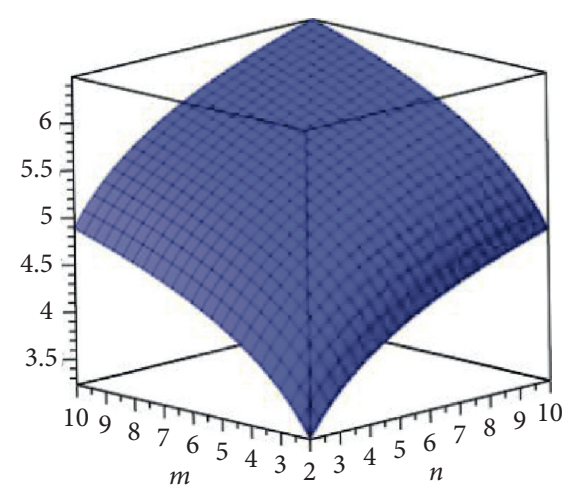

(a)

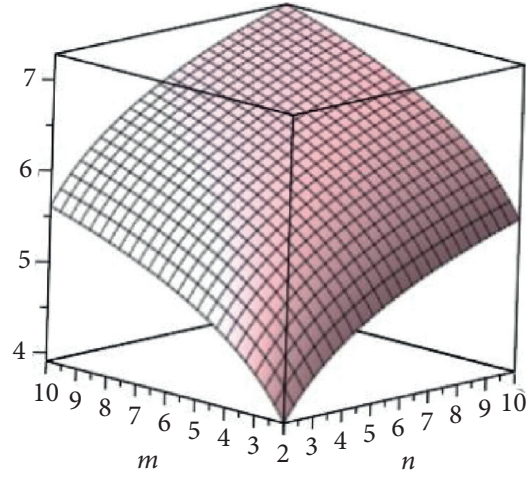

(b)

Figure 12: (a) The $A B C_{4}$ entropy; (b) the $\mathrm{GA}_{5}$ entropy. 
TABle 12: Comparison of Randić entropies for $\mathrm{Si}_{2} \mathrm{C}_{3}-I I[m, n]$.

\begin{tabular}{llllr}
\hline$[m, n]$ & $E_{R_{1}}$ & $E_{R_{-1}}$ & $E_{R_{1 / 2}}$ & $E_{R_{-1 / 2}}$ \\
\hline$[2,2]$ & 2.44 & 0.60 & 2.01 & 1.14 \\
{$[3,3]$} & 2.99 & 1.02 & 2.46 & 1.56 \\
{$[4,4]$} & 3.22 & 1.32 & 2.76 & 1.84 \\
{$[5,5]$} & 3.44 & 1.55 & 2.98 & 2.19 \\
{$[6,6]$} & 3.61 & 1.73 & 3.29 & 2.05 \\
{$[7,7]$} & 3.76 & 1.88 & 3.41 & 2.36 \\
{$[8,8]$} & 3.88 & 2.01 & 3.52 & 2.48 \\
{$[9,9]$} & 3.99 & 2.11 & 3.62 & 2.59 \\
{$[10,10]$} & 4.09 & 2.21 & & 2.68 \\
\hline
\end{tabular}

TABLE 13: Comparison of $E_{A B C}, E_{\mathrm{GA}}, E_{M_{1}}$, and $E_{M_{2}}$ entropies for $\mathrm{Si}_{2} \mathrm{C}_{3}-I I[m, n]$.

\begin{tabular}{lcccc}
\hline$[m, n]$ & $E_{A B C}$ & $E_{\mathrm{GA}}$ & $E_{M_{1}}$ & $E_{M_{2}}$ \\
\hline$[2,2]$ & 1.43 & 1.80 & 2.34 & 2.78 \\
{$[3,3]$} & 1.85 & 2.17 & 3.07 & \\
{$[4,4]$} & 2.13 & 2.42 & 3.28 & 3.92 \\
{$[5,5]$} & 2.34 & 2.61 & 3.46 & \\
{$[6,6]$} & 2.52 & 2.77 & 3.60 & 3.44 \\
{$[7,7]$} & 2.66 & 2.90 & 3.72 & 3.61 \\
{$[8,8]$} & 2.78 & 3.01 & 3.83 & 3.88 \\
{$[9,9]$} & 2.88 & 3.11 & 3.92 & 3.99 \\
{$[10,10]$} & 2.98 & 3.20 & & 4.09 \\
\hline
\end{tabular}

TABLE 14: Comparison of $E_{\mathrm{HM}}, E_{F}, E_{\mathrm{AZI}}$, and $E_{J}$ entropies for $\mathrm{Si}_{2} \mathrm{C}_{3}-I I[m, n]$.

\begin{tabular}{lcccc}
\hline$[m, n]$ & $E_{\mathrm{HM}}$ & $E_{F}$ & $E_{\mathrm{AZI}}$ & 2.56 \\
\\
{$[2,2]$} & 2.99 & 2.73 & 3.03 & \\
{$[3,3]$} & 3.51 & 3.22 & 3.33 & \\
{$[4,4]$} & 3.81 & 3.52 & 3.55 & 2.16 \\
{$[5,5]$} & 4.04 & 3.70 & 3.72 & \\
{$[6,6]$} & 4.21 & 3.92 & 3.87 & \\
{$[7,7]$} & 4.36 & 4.06 & 3.99 & 2.61 \\
{$[8,8]$} & 4.48 & 4.19 & 4.10 & 2.76 \\
{$[9,9]$} & 4.59 & 4.29 & 4.19 & 3.01 \\
{$[10,10]$} & 4.69 & 4.39 & & 3.10 \\
\hline
\end{tabular}

TABLE 15: Comparison of the redefined Zagreb entropies for $\mathrm{Si}_{2} \mathrm{C}_{3}-I I[m, n]$.

\begin{tabular}{lccc}
\hline$[m, n]$ & $E_{\mathrm{ReZG}_{1}}$ & $E_{\mathrm{ReZG}_{2}}$ & $E_{\mathrm{ReZG}_{3}}$ \\
\hline$[2,2]$ & 1.56 & 1.42 & 3.18 \\
{$[3,3]$} & 1.93 & 1.93 & 3.67 \\
{$[4,4]$} & 2.19 & 2.24 & 3.98 \\
{$[5,5]$} & 2.39 & 2.46 & 4.20 \\
{$[6,6]$} & 2.54 & 2.64 & 4.38 \\
{$[7,7]$} & 2.68 & 2.79 & 4.53 \\
{$[8,8]$} & 2.80 & 2.92 & 4.65 \\
{$[9,9]$} & 2.90 & 3.03 & 4.76 \\
{$[10,10]$} & 2.99 & 3.13 & 4.86 \\
\hline
\end{tabular}


TABLE 16: Comparison of the $A B C_{4}$ and $\mathrm{GA}_{5}$ entropies for $\mathrm{Si}_{2} \mathrm{C}_{3}-I I[m, n]$.

\begin{tabular}{lcr}
\hline$[m, n]$ & $E_{A B C_{4}}$ & $E_{\mathrm{GA}_{5}}$ \\
\hline$[2,2]$ & 1.34 & 1.64 \\
{$[3,3]$} & 1.73 & 2.05 \\
{$[4,4]$} & 1.99 & 2.32 \\
{$[5,5]$} & 2.20 & 2.53 \\
{$[6,6]$} & 2.36 & 2.70 \\
{$[7,7]$} & 2.50 & 2.84 \\
{$[8,8]$} & 2.62 & 2.96 \\
{$[9,9]$} & 2.72 & 3.06 \\
{$[10,10]$} & 2.81 & 3.16 \\
\hline
\end{tabular}

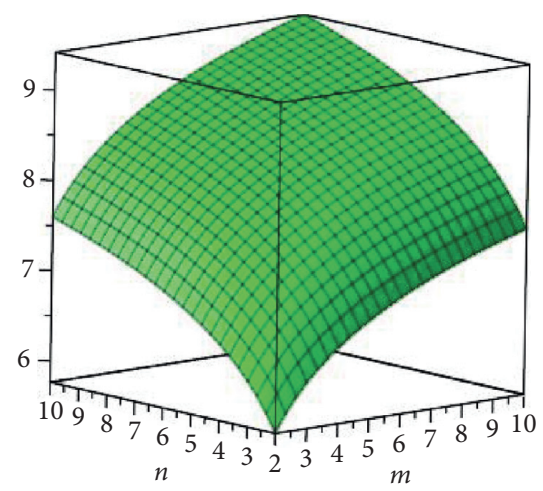

(a)

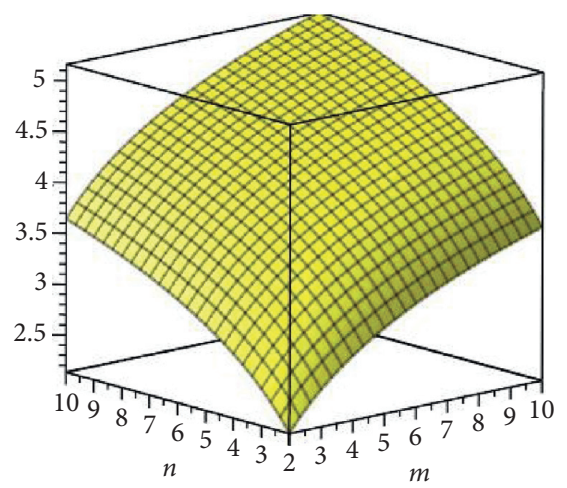

(b)

Figure 13: (a) Randić entropy for $\alpha=1$; (b) Randić entropy for $\alpha=-1$.

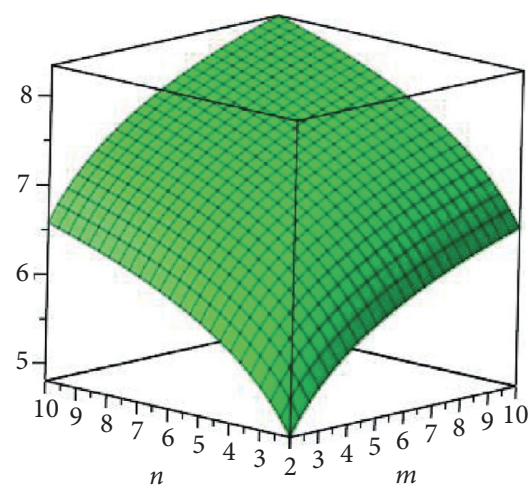

(a)

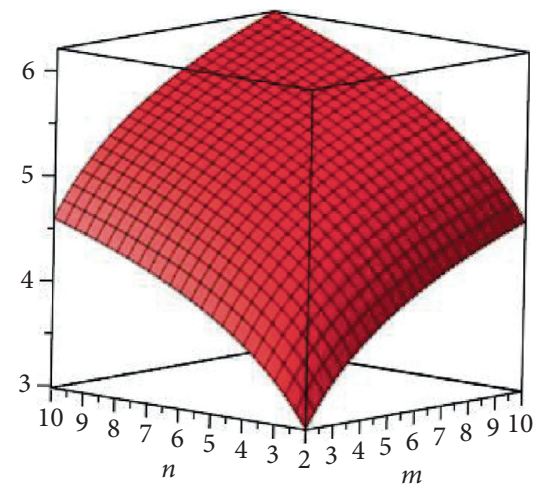

(b)

Figure 14: (a) Randić entropy for $\alpha=(1 / 2)$; (b) Randić entropy for $\alpha=(-1 / 2)$.

for $\mathrm{Si}_{2} \mathrm{C}_{3}-I I[m, n]$. Also, we produce Tables $12-16$ with the help of Matlab for small estimations of $m, n$. From tables, we can note that all the evaluation of entropy is in growing request as the values of parameters are expanded. These estimations are very helpful for the chemist to analyse chemical properties of the structures. The graphical representation of computed findings is demonstrated in Figures 13-20 for certain measurements of $m, n$. 


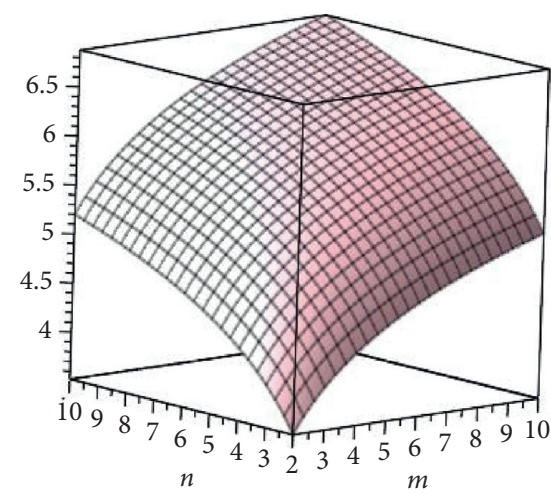

(a)

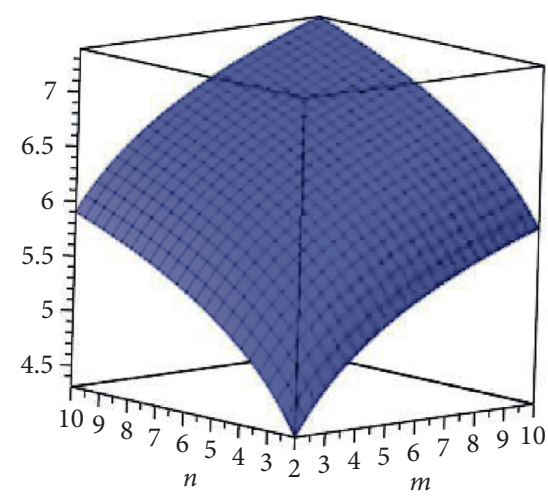

(b)

Figure 15: (a) The atom bond connectivity entropy; (b) the geometric arithmetic entropy.

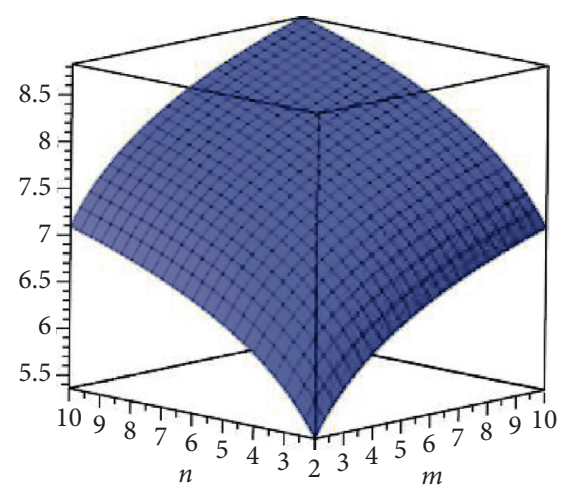

(a)

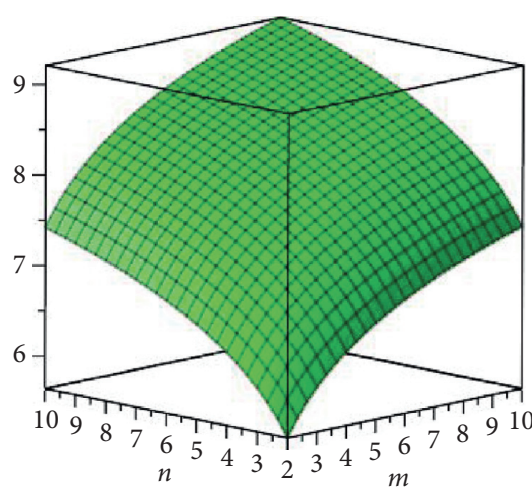

(b)

FIgURe 16: (a) The first Zagreb entropy; (b) the second Zagreb entropy.

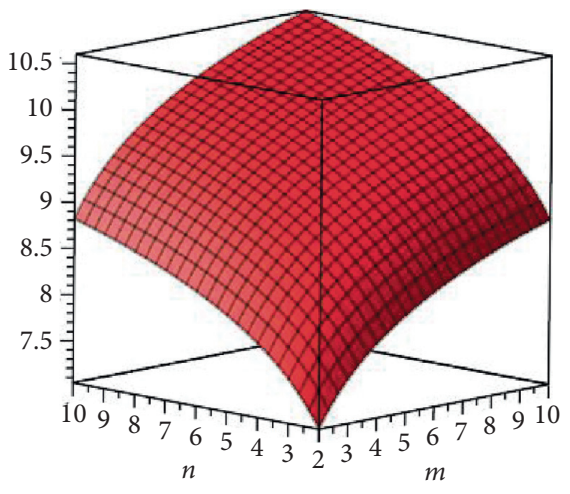

(a)

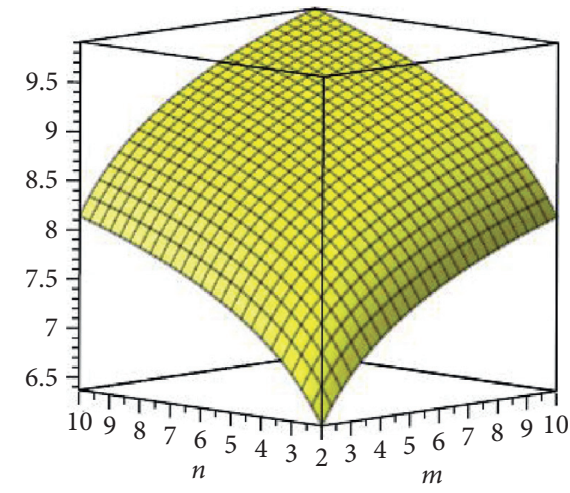

(b)

Figure 17: (a) The hyper-Zagreb entropy; (b) the forgotten entropy. 


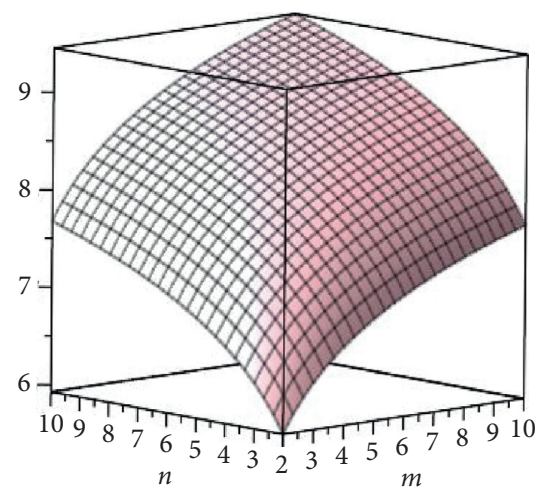

(a)

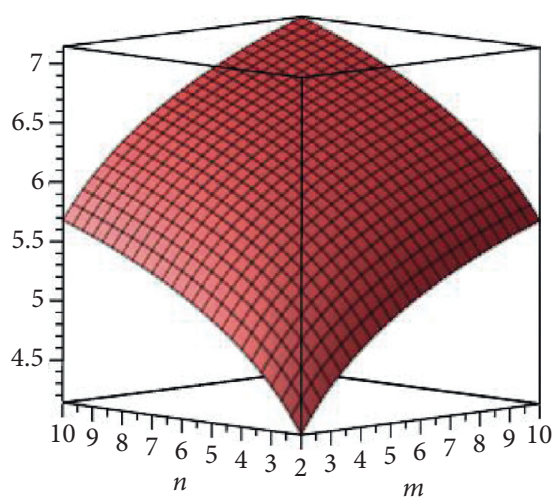

(b)

Figure 18: (a) The augmented Zagreb entropy; (b) the Balaban entropy.

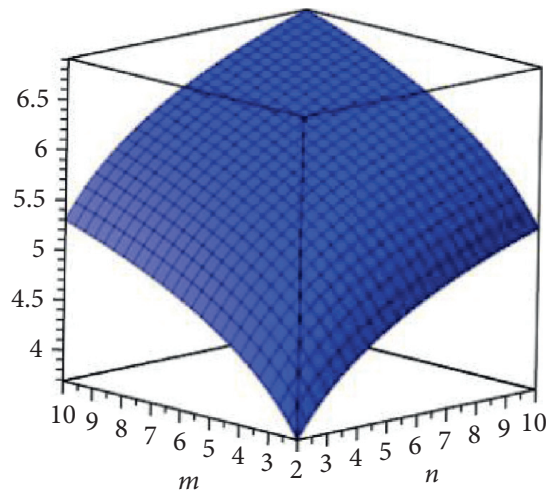

(a)

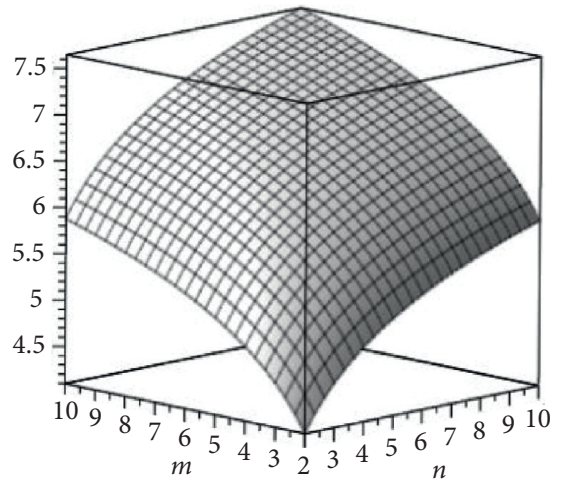

(b)

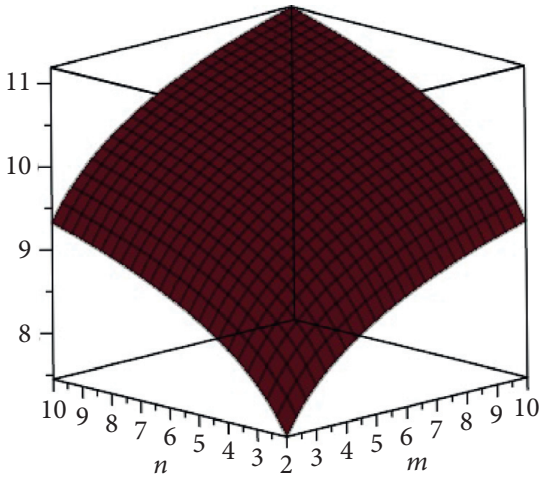

(c)

Figure 19: (a) The redefined first Zagreb entropy; (b) the redefined second Zagreb entropy; (c) the redefined third Zagreb entropy.

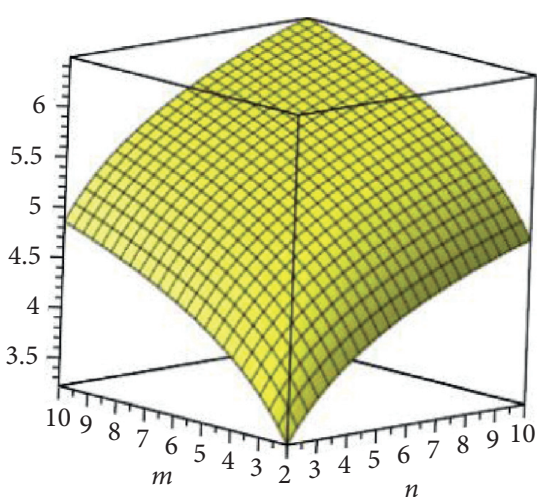

(a)

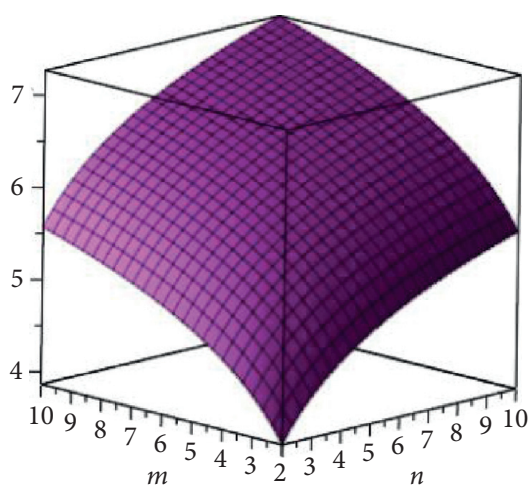

(b)

Figure 20: (a) The $A B C_{4}$ entropy; (b) the $\mathrm{GA}_{5}$ entropy.

\section{Conclusion}

In this study, we considered the molecular structure of silicon carbide $\mathrm{Si}_{2} \mathrm{C}_{3}-I[m, n]$ and $\mathrm{Si}_{2} \mathrm{C}_{3}-I I[m, n]$. We estimated degree-based indices like general Randić index, $A B C$ index, GA index, Zagreb type indices, forgotten index,
hyper-Zagreb index, AZI index, Balaban index, redefined Zagreb indices, fourth atom bond connectivity index, and fifth geometric arithmetic index. Afterwards, we extend our computation towards the estimation of corresponding entropies of aforementioned degree-based indices. We compared these estimations both numerically and graphically. 
These entropies associate particular physicochemical characteristics like distortion, stability, melting points, and strain energy of chemical compounds. The mathematical findings for these graphs are helpful for the chemist to understand the biochemical utilization of these structures.

\section{Data Availability}

The data used to support the findings of this study are cited at relevant places within the text as references.

\section{Conflicts of Interest}

The authors declare that they have no conflicts of interest.

\section{Authors' Contributions}

All authors contributed equally to this work.

\section{Acknowledgments}

This research was supported by Domestic Visiting and Study Program for Outstanding Young Backbone Talents in Universities (Grant no. gxgnfx2021165).

\section{References}

[1] M. Randic, "Characterization of molecular branching," Journal of the American Chemical Society, vol. 97, no. 23, pp. 6609-6615, 1975.

[2] I. Gutman, B. Ruscic, N. Trinajstić, and C. F. Wilcox Jr, "Graph theory and molecular orbitals. XII. Acyclic polyenes," The Journal of Chemical Physics, vol. 62, no. 9, pp. 3399-3405, 1975.

[3] I. Gutman and K. C. Das, "The first Zagreb index 30 years after," MATCH Communications in Mathematical and in Computer Chemistry, vol. 50, pp. 83-92, 2004.

[4] G. H. Shirdel, H. RezaPour, and A. M. Sayadi, "The hyper zagreb index of graph operations," Iranian Journal of Mathematical Chemistry, vol. 4, no. 2, pp. 213-220, 2013.

[5] B. Furtula and I. Gutman, "A forgotten topological index," Journal of Mathematical Chemistry, vol. 53, no. 4, pp. 1184-1190, 2015.

[6] B. Furtula, A. Graovac, and D. Vukičević, "Augmented zagreb index," Journal of Mathematical Chemistry, vol. 48, no. 2, pp. 370-380, 2010.

[7] A. T. Balaban, "Highly discriminating distance-based topological index," Chemical Physics Letters, vol. 89, no. 5, pp. 399-404, 1982.

[8] A. T. Balaban and L. V. Quintas, "The smallest graphs, trees, and 4-trees with degenerate topological index," Journal of Mathematical Chemistry, vol. 14, pp. 213-233, 1983.

[9] P. S. Ranjini, V. Lokesha, and A. Usha, "Relation between phenylene and hexagonal squeez using harmonic index," International Journal of Graph Theory, vol. 1, pp. 116-121, 2013.

[10] A. Ghorbani and M. A. Hosseinzadeh, "Computing $A B C_{4}$ index of nanostar dendrimers," Optoelectronics and Advanced Materials-Rapid Communications, vol. 4, pp. 1419-1422, 2010.

[11] A. Graovac, M. Ghorbani, and M. A. Hosseinzadeh, "Computing fifth geometric-arithmetic index for nanostar dendrimers," Journal of Mathematical Nanoscience, vol. 1, pp. 33-42, 2011.
[12] M. K. Siddiqui, M. Imran, and A. Ahmad, "On zagreb indices, zagreb polynomials of some nanostar dendrimers," Applied Mathematics and Computation, vol. 280, pp. 132-139, 2016.

[13] M. K. Siddiqui, M. Naeem, N. A. Rahman, and M. Imran, "Computing topological indices of certain networks," Journal of Optoelectronics and Advanced Materials, vol. 18, no. 9-10, pp. 884-892, 2016.

[14] D. Amic, D. Beslo, B. Lucic, S. Nikolic, and N. Trinajstić, "The vertex-connectivity index revisited," Journal of Chemical Information and Computer Sciences, vol. 38, pp. 819-822, 1998.

[15] K. Anand and G. Bianconi, "Entropy measures for networks: toward an information theory of complex topologies," Physical Review. E, Statistical, Nonlinear, and Soft Matter Physics, vol. 80, no. 4, pp. 045102-045155, 2009.

[16] C. E. Shannon, "A mathematical theory of communication," Bell System Technical Journal, vol. 27, no. 3, pp. 379-423, 1948.

[17] P. Ali, S. A. K. Kirmani, O. Al Rugaie, and F. Azam, "Degreebased topological indices and polynomials of hyaluronic acidcurcumin conjugates," Saudi Pharmaceutical Journal, vol. 28, no. 9, pp. 1093-1100, 2020.

[18] F. Yin, M. Numan, S. I. Butt, A. Aslam, and A. Kausar, "Topological indices of derived networks of benzene ring embedded in P -type surface on 2D," Journal of Chemistry, vol. 2021, Article ID 6614840, 11 pages, 2021.

[19] O. C. Havare and A. K. Havare, "Computation of the forgotten topological index and co-index for carbon base nanomaterial," Polycyclic Aromatic Compounds, vol. 8, pp. 1-13, 2020.

[20] S. Cao and M. Dehmer, "Degree-based entropies of networks revisited," Applied Mathematics and Computation, vol. 261, pp. 141-147, 2015.

[21] M. Dehmer and A. Mowshowitz, "A history of graph entropy measures," Information Sciences, vol. 181, no. 1, pp. 57-78, 2011.

[22] S. Manzoor, M. K. Siddiqui, and S. Ahmad, "On entropy measures of molecular graphs using topological indices," Arabian Journal of Chemistry, vol. 13, no. 8, pp. 6285-6298, 2020.

[23] N. Rashevsky, "Life, information theory, and topology," Bulletin of Mathematical Biophysics, vol. 17, no. 3, pp. 229235, 1955.

[24] Z. Chen, M. Dehmer, and Y. Shi, "A note on distance-based graph entropies," Entropy, vol. 16, no. 10, pp. 5416-5427, 2014.

[25] G. Bianconi, "The entropy of randomized network ensembles," Europhysics Letters, vol. 81, no. 2, pp. 28-35, 2008.

[26] W. Gao, M. Imran, M. K. Siddiqui, M. Naeem, and F. Jamil, "Molecular description of copper (I) oxide and copper (II) oxide," Quimica Nova, vol. 41, pp. 874-879, 2018.

[27] Z.-Q. Cai, A. Rauf, M. Ishtiaq, and M. K. Siddiqui, "On vedegree and ev-degree based topological properties of silicon carbide Si2C3-II [p, q]," Polycyclic Aromatic Compounds, vol. 10, pp. 1-15, 2020.

[28] S. M. Kang, M. K. Siddiqui, N. A. Rehman, M. Naeem, and M. H. Muhammad, "Topological properties of 2-dimensional silicon-carbons,” IEEE Access, vol. 6, pp. 59362-59373, 2018.

[29] G. V. Rajasekharaiah and U. P. Murthy, "Hyper-Zagreb indices of graphs and its applications," Journal of Algebra Combinatorics Discrete Structures and Applications, vol. 8, no. 1, pp. 9-22, 2021.

[30] D. Janezic, A. Milicevic, S. Nikolic, N. Trinajstic, and D. Vukicevic, "Zagreb indices: extension to weighted graphs representing molecules containing heteroatoms," Croatica Chemica Acta, vol. 80, no. 3-4, pp. 541-545, 2007. 
[31] M. Cai, R. Y. K. Du Hai-Feng, and W. Marcus, "A new network structure entropy based node difference and edge difference," Acta Physica Sinica, vol. 60, pp. 110-123, 2011.

[32] Y.-H. Xiao, W.-T. Wu, H. Wang, M. Xiong, and W. Wang, "Symmetry-based structure entropy of complex networks," Physica A: Statistical Mechanics and Its Applications, vol. 387, no. 11, pp. 2611-2619, 2008. 